The Astrophysical Journal, 476:572-588, 1997 February 20

(C) 1997. The American Astronomical Society. All rights reserved. Printed in U.S.A.

\title{
STATISTICAL EFFECTS OF DOPPLER BEAMING AND MALMQUIST BIAS ON FLUX-LIMITED SAMPLES OF COMPACT RADIO SOURCES
}

\author{
Matthew L. Lister AND Alan P. Marscher \\ Department of Astronomy, Boston University, 725 Commonwealth Avenue, Boston, MA 02215 \\ Received 1995 November 3; accepted 1996 September 16
}

\begin{abstract}
We examine the effects of Doppler beaming on flux-limited samples of compact radio sources representative of relativistic jets found in active galactic nuclei (AGNs). We expand upon past studies by incorporating a luminosity function and redshift distribution for the parent population and by allowing the unbeamed synchrotron luminosity $L$ of a relativistic jet to be related to its bulk Lorentz factor $(\Gamma)$. These enhancements allow us to compare observable parameters other than simply apparent velocity with the data. The predictions of $L-\Gamma$-independent (LGI) models are compared to those of a $L-\Gamma-$ dependent (LGD) scenario in which the Lorentz factor and luminosity are related by the form $L \propto \Gamma^{\xi}$. This is accomplished using Monte Carlo simulations, where we compare the predicted flux density, redshift, monochromatic emitted luminosity, and apparent velocity distributions of flux-limited samples to the Caltech-Jodrell Bank sample of bright, flat-spectrum, radio core-dominated AGNs (CJ-F).

The LGI model predictions are consistent with the CJ-F data if we adopt parent Lorentz factor distributions of the form $N(\Gamma) \propto \Gamma^{a}$, where $-1.5 \lesssim a \lesssim-1.75$, or, alternatively $N(\Gamma) \propto(\Gamma-1)^{a}$, where $-0.5 \lesssim a \lesssim-1$. These models reproduce, via selection effects, a deficit of sources having both low apparent velocity $\left(\beta_{\text {app }}\right)$ and high monochromatic emitted luminosity $(P)$ seen in the CJ-F sample, as reported by Vermeulen in 1995.

We examine two possible cases for the LGD scenario, the first of which employs a positive correlation between unbeamed synchrotron luminosity and Lorentz factor (the LGC model), and the second of which employs an anticorrelation (the LGA model). The LGA models do not predict enough low- $P$ sources to be consistent with the CJ-F data and do not reproduce the $P$ versus $\beta_{\text {app }}$ envelope. The predictions of the best-fit LGC model, on the other hand, are very similar to our best-fit LGI models and provide as good fits to the CJ-F data, with the important exception that very few high-viewing angle sources are predicted. This in conflict with the large fraction (11\%) of radio galaxies present in the CJ-F sample, whose jet axes are predicted by the unified AGN model to be at angles greater than $\sim 45^{\circ}$ to the line of sight.

At present, the observational data on the CJ-F sample are otherwise insufficient to distinguish between the LGI and LGC models. Our simulations indicate, however, that the LGC models predict a larger number of high $(\delta>20)$ Doppler factor sources in flux-limited samples. Furthermore, the predicted median variability timescale of the high $\left(\beta_{\mathrm{app}}>10 \mathrm{~h}^{-1}\right)$ objects is only $\sim 20$ times faster than the low $\beta_{\text {app }}<2.5 h^{-1}$ objects in the LGC models, whereas the LGI models predict a ratio of $\sim 200$.

We find that all of our models predict a very large parent population for the CJ-F sample: on the order of $10^{7}-10^{7.7}$ objects are required to produce 293 objects with flux densities greater than $350 \mathrm{mJy}$ for the LGC and LGI models, respectively. This translates into parent population space densities on the order of (1.3-5.9) $\times 10^{-5} \mathrm{Mpc}^{-3}$ for $H_{0}=65 \mathrm{~km} \mathrm{~s}^{-1} \mathrm{Mpc}^{-1}$ and $q_{0}=0$.
\end{abstract}

Subject headings: galaxies: active — galaxies: jets — methods: statistical — quasars: general

\section{INTRODUCTION}

It is now clear that the study of active galactic nuclei (AGNs), like many other areas in astronomy, is strongly affected by the biases inherent in flux-limited sampling. In survey studies of stars and galaxies, the effects of a lower flux cutoff have been well established and are relatively straightforward to model by assuming simple power-law luminosity functions (LFs) for the source populations. Such methods are not as easily applied to AGNs, owing to orientation biases such as the Doppler beaming of nonthermal emission caused by relativistic motions of the radiating plasma (in the form of jets). This phenomenon, which causes the apparent flux of a jet to be boosted along directions nearly parallel to the flow, has been predicted to affect to varying degrees the apparent LFs of radio galaxies, quasars, and other types of AGNs (Urry \& Shafer 1984; Urry \& Padovani 1991).
Flux-limited samples of AGNs are thought to contain a particularly complicated form of selection bias, since they are expected to include not only sources of high intrinsic luminosity but also lower luminosity sources whose emission is Doppler boosted by virtue of their orientation with respect to the observer. The exact composition of such a sample is therefore highly dependent on certain aspects of the parent population. These include the intrinsic dispersion of jet Lorentz factors, the distribution of jet orientations, and the intrinsic (nonboosted) LF.

The basic properties of such a Doppler-boosted sample have been explored in several theoretical papers (see, e.g., Urry \& Shafer 1984; Urry \& Padovani 1991; Vermeulen \& Cohen 1994), with the general result being that such a sample will contain mostly objects aligned close to the line of sight (the so-called Doppler favoritism effect). By virtue of the orientations and relativistic speeds of their jets, these 
objects are also expected to display apparent superluminal motion; the expected apparent velocity distributions for flux-limited samples have been investigated by Vermeulen \& Cohen (1994) and Vermeulen (1995).

Although the predictions of these models have been shown to be generally consistent with observations, they have not included both an intrinsic luminosity function and redshift distribution, so that the combined effects of Malmquist and beaming biases on flux-limited samples have not yet been explored fully. Another important aspect of these past studies is that they have all assumed only a basic scenario, in which the intrinsic, unbeamed LF of the jet population is independent of any of the physical properties of the sources. However, physical models of relativistic jets, such as those based on hydrodynamics (see, e.g., Blandford \& Rees 1974), predict the synchrotron luminosity $L$ to be highly dependent upon the Lorentz factor $\Gamma$ (Marscher 1980). Here, we adopt a power-law dependence $L \propto \Gamma^{\xi}$, where $\xi$ is a free parameter.

The predicted properties of flux-limited samples based on a $L$ - $\Gamma$-dependent (LGD) model are expected to differ from those of Padovani \& Urry (1992) owing to the dependence of both the Doppler boosting and intrinsic luminosity on $\Gamma$. In this paper, we investigate the properties of flux-limited samples based on the basic $L-\Gamma$-independent (LGI) and LGD model scenarios using both analytical and Monte Carlo techniques and compare the results to an existing observational data set selected on the basis of compact (parsec-scale) flux density (the Caltech-Jodrell Bank flatspectrum AGN sample; Taylor et al. 1996). For our comparisons, we concentrate primarily on the apparent velocity, flux density, monochromatic emitted luminosity, and redshift distributions, the latter three of which have yet to be explored fully in the literature for flux-limited, Dopplerboosted samples. We also examine possible correlations between other observable source quantities such as monochromatic emitted luminosity (also sometimes referred to as beamed power), variability timescale, viewing angle, and Doppler factor.

\section{JET POPULATIONS HAVING A SINGLE LORENTZ FACTOR}

\subsection{No Cosmological Evolution}

In order to illustrate the statistical effects of relativistic beaming and cosmological luminosity evolution in isolation, we begin by examining a very simple case in which analytical solutions exist for the redshift and apparent velocity distributions of flux-limited samples. Our canonical jet population is assumed to have (1) random jet orientations, (2) a single Lorentz factor $\Gamma$, (3) a single spectral index $\alpha=0\left(S \propto v^{\alpha}\right)$, (4) an intrinsic (unbeamed) jet synchrotron luminosity $L$ that contains no contribution from extended (lobe) structure and is independent of $\Gamma$ and all other parameters, and (5) a constant comoving space density distributed between $0<z<4$. The intrinsic luminosity function in this model does not evolve with redshift and is taken to be of the form

$$
\phi(L)=\phi_{0} L^{g}, \quad L_{1} \leq L<\infty .
$$

The observed (beamed) luminosity function will be different from this intrinsic LF owing to the effects of relativistic beaming. These effects have been studied in detail by Urry \& Shafer (1984) and Urry \& Padovani (1991). In particular, they find that the beamed and unbeamed LFs have the same slope at high luminosities.

The measured flux density is (for the purpose of deriving analytical expressions, we will ignore the emission from the presumed counterjet)

$$
S=\frac{L \delta^{p}}{4 \pi d_{l}^{2}(z)}(1+z)^{1+\alpha}
$$

where $d_{l}(z)$ is the luminosity distance, and the exponent $p$ has the value $2-\alpha$ for steady state or time-averaged jets. The Doppler-boosting factor is $\delta=\Gamma^{-1}(1-\beta \cos \theta)^{-1}$, where $\theta$ is the angle between the line of sight and the jet axis, and $\beta=\left(1-\Gamma^{-2}\right)^{1 / 2}$. The maximum value of $\delta$ is $\delta_{\max }=$ $\left[\Gamma-\left(\Gamma^{2}-1\right)^{1 / 2}\right]^{-1} \approx 2 \Gamma$ for large $\Gamma$, while $\delta_{\min }=1 / \Gamma$. In formulae of the next section, we will for convenience define

$$
k(z) \equiv \frac{1}{4 \pi}(1+z)^{1+\alpha} .
$$

\subsection{Redshift Distribution}

For a given flux cutoff $S$, the expression for $n(z) d z$ will take on different forms for various ranges of $z$. This is due to the fact that the beamed LF has different slopes in the regions $L_{1} \delta_{\min }^{p}<L<L_{1} \delta_{\max }^{p}$ and $L_{1} \delta_{\max }^{p}<L<\infty$ (see Urry \& Padovani 1991 for a more detailed discussion of beamed LFs).

Region $1: d_{l}(z) \leq\left[L_{1} k(z) S^{-1} \delta_{\min }^{p}\right]^{1 / 2}$

In this region, the entire range of the LF can be seen down to the lower limit $L_{1}$, so we have

$$
n(z) d z=N_{\text {tot }} \frac{d V}{d z} d z .
$$

The comoving volume element for $q_{0}=0$ is

$$
\frac{d V}{d z}=\frac{c^{3} z^{2}(1+z / 2)^{2}}{H_{0}^{3}(1+z)^{3}},
$$

where $c$ is the speed of light and $H_{0}$ is the Hubble constant. Throughout this paper, we use $q_{0}=0$ and $h=0.65$, where $h$ is the Hubble constant expressed in units of $100 \mathrm{~km} \mathrm{~s}^{-1} \mathrm{Mpc}^{-1}$.

Region 2: $\left[L_{1} k(z) \dot{S}^{-1} \delta_{\min }^{p}\right]^{1 / 2}<d_{l}(z) \leq\left[L_{1} k(z) S^{-1} \delta_{\max }^{p}\right]^{1 / 2}$

In this region, the weakest sources in the parent population, which have $L=L_{1}$ and $\delta=\delta_{\min }$, can no longer be seen. We have

$$
\begin{aligned}
& n(z) d z= \\
& \quad N_{\text {tot }} \frac{d V}{d z} d z\left[\int_{\Gamma^{-1}}^{\delta_{1}} \int_{L_{\min }}^{\infty} \Phi(L) P(\delta) d \delta d L+\int_{\delta_{1}}^{\delta_{\max }} P(\delta) d \delta\right],
\end{aligned}
$$

where

$$
\delta_{1} \equiv\left[S d_{l}^{2}(z) / L_{1} k(z)\right]^{1 / p},
$$

$P(\delta)=\left(\delta^{2} \beta \Gamma\right)^{-1}$ is the probability density function for $\delta$, $\Phi(L)$ is the normalized luminosity function, and $L_{\min }=$ $S d_{l}^{2}(z) /\left(k(z) \delta^{p}\right)$.

Region 3: $d_{l}(z)>\left[L_{1} k(z) S^{-1} \delta_{\max }^{p}\right]^{1 / 2}$

In this region, the sources in the parent population that have $L=L_{1}$ can no longer be seen, so

$$
n(z) d z=N_{\text {tot }} \frac{d V}{d z} d z \int_{\Gamma^{-1}}^{\delta_{\max }} \int_{L_{\min }}^{\infty} \Phi(L) P(\delta) d \delta d L .
$$


The above expressions can be solved analytically; the predicted $z$-distributions for several different sets of parameters are plotted in Figure 1. The left- and right-hand columns have LF slopes of $g=-2.48$ and $g=-3.5$, respectively, and $L_{1}=10^{24} \mathrm{~W} \mathrm{~Hz}^{-1}$. The Lorentz factor increases progressively for each row, with the first row $(\Gamma=1)$ corresponding to the unbeamed case in which all sources have nonrelativistic jet speeds. To facilitate comparisons, the curves have all been normalized to the same peak value. In each panel we have plotted the predicted distributions for two different flux cutoffs, one at $0.35 \mathrm{Jy}$ (solid line) and the other at $3.5 \mathrm{Jy}$ (dashed line).

It is apparent from this figure that relativistic beaming plays a large role in determining the shape of the redshift distribution, primarily at high $z$. In this region, the volume element is large, which gives a larger probability of finding highly aligned sources that are undergoing the maximum amount of Doppler boosting. By increasing the Lorentz factor of the population, $\delta_{\max }$ increases, so that a higher number of these high-redshift sources make it into a fluxlimited sample. The tail end of the $z$-distribution is thus raised, and the peak of the distribution moves to slightly higher $z$.

The slope of the intrinsic LF also has an impact on the $z$-distribution, which is more pronounced in the lower $\Gamma$ models. This is simply a reflection of the fact that the maximum Doppler boost is lower for these models, so that the intrinsic LF shape is a more important factor than relativistic beaming in determining whether a source will be included in the sample.

\subsection{Pure Luminosity Evolution}

Studies of the radio galaxy luminosity function (see, e.g., Padovani \& Urry 1992; Dunlop \& Peacock 1990) have shown that it evolves with redshift. It is best described by a pure luminosity evolution model, in which sources increase in brightness with lookback time but maintain a constant comoving density.

In their study of the FR II radio galaxy LF at $2.7 \mathrm{GHz}$, Padovani \& Urry (1992) characterize this evolution using a form $L(z)=L(z=0) \exp [T(z) / \tau]$, where $T(z)$ is the lookback time and $\tau$ is the timescale of evolution in units of the Hubble time. A low value of $\tau$ thus corresponds to a large amount of evolution, and vice versa.

Using data from the 2 Jy Wall \& Peacock (1985) sample, these authors obtain a good fit to the LF using a double power law, with a break at $10^{26 \pm 0.2} h^{-2} \mathrm{~W} \mathrm{~Hz}^{-1}$. The slopes before and after the break are $-2.48 \pm 0.15$ and $-3.9 \pm 0.7$, respectively. The best-fit value for the evolution parameter is $\tau=0.26$, with a $1 \sigma$ confidence interval [0.16, 1.0] (updated values from Urry \& Padovani 1995). We will hereafter refer to this LF fit as the PULF.

Pure luminosity evolution can be implemented in our model simply by replacing $L_{1}$ with $L_{1}(z)$ in the previous equations for $n(z)$. We plot predicted distributions for two evolution models with $g=-2.48$ and $L_{1}=10^{24} \mathrm{~W} \mathrm{~Hz}^{-1}$ in Figure 2. The left-hand column is for $\tau=0.26$, and in the right-hand column, we show the effects of changing $\tau$ to 0.2 .

The effects of evolution are most pronounced at high redshift, since these sources will have much higher intrinsic luminosities. If we compare the left-hand columns of Figures 1 and 2 , the evolution models contain a much higher relative number of high-redshift sources, as expected. The value of $\tau$ also has a large effect on the position of the peak and the high-redshift tail of the $z$ distributions.

\subsection{Apparent Velocity Distributions}

The apparent velocity distributions of orientation-biased samples having single values of Lorentz factor have been investigated by Vermeulen \& Cohen (1994), who find that they are generally peaked at $\beta_{\text {app }} \simeq \beta_{\text {app, } \max } \simeq \Gamma$, with numbers decreasing toward lower $\beta_{\text {app }}$. As analytical expressions for $N\left(\beta_{\text {app }}\right)$ cannot be obtained for a model such as ours that includes an LF, we have performed Monte Carlo simulations and find that the LF parameters have very little effect on the $\beta_{\text {app }}$ distributions. As we will describe in $\S 5$, the $\beta_{\text {app }}$ distribution for a flux-limited sample in which the unbeamed synchrotron luminosity is independent of $\Gamma$ is determined almost entirely by the intrinsic Lorentz factor distribution of the parent population.

\section{OBSERVATIONAL DATA}

Since the canonical sources in our models are selected on the basis of jet (beamed) flux only, it is important to make comparisons with a complete sample selected on the basis of high-frequency flux density. This choice serves to minimize the contribution of any steep-spectrum extended structure to the observed flux density. The best such sample in the literature is the Caltech-Jodrell flat-spectrum AGN sample (CJ-F: Taylor et al. 1996). It consists of all AGNs with (1) declination $(1950.0) \geq 35^{\circ}$, (2) Galactic latitude $|b| \geq 10^{\circ}$, (3) total $4850 \mathrm{MHz}$ flux density $\geq 0.35 \mathrm{Jy}$, and (4) spectral index between 1400 and $4850 \mathrm{MHz}$ flatter than -0.5 . In total, 293 sources meet these criteria, of which currently 192 have measured redshifts and 81 have multiepoch VLBI data (Vermeulen 1995).

The distribution of apparent velocities for the CJ-F sample (omitting 21 sources for which there are only upper limits) is plotted in the top left panel of Figure 3. It is peaked at low $\beta_{\text {app }}$ and thus appears to be in conflict with the predictions of the simple single-valued Lorentz factor model described in § 2. Indeed, Vermeulen \& Cohen (1994) rule out the single-valued Lorentz factor distribution, unless there are substantial differences between the pattern and bulk flow Lorentz factors in jets. These differences will be discussed more fully in $\S 4.2$. We turn now to models in which the parent populations have a distribution of Lorentz factors.

\section{MODELS WITH A DISTRIBUTION OF LORENTZ FACTORS: COMPONENTS AND ASSUMPTIONS}

As analytical solutions for the redshift and $\beta_{\text {app }}$ distributions can be found only for the single-valued Lorentz factor case, in this section we make extensive use of Monte Carlo simulations to explore the class of models in which there is a distribution of Lorentz factors. Our simulation procedure is to create iteratively canonical sources until we obtain 293 objects with flux densities $\geq 0.35 \mathrm{Jy}$. The total size of the parent population is therefore a function of the LF parameters and Lorentz factor distribution. A description of our choices of parameters follows.

\subsection{Luminosity Function}

Unfortunately, very little is known about the intrinsic (unbeamed) LF of the CJ-F parent population, as it is extremely difficult to disentangle the various effects of relativistic beaming and evolution on the sample. In this paper, our canonical jet population follows the parameters of the 


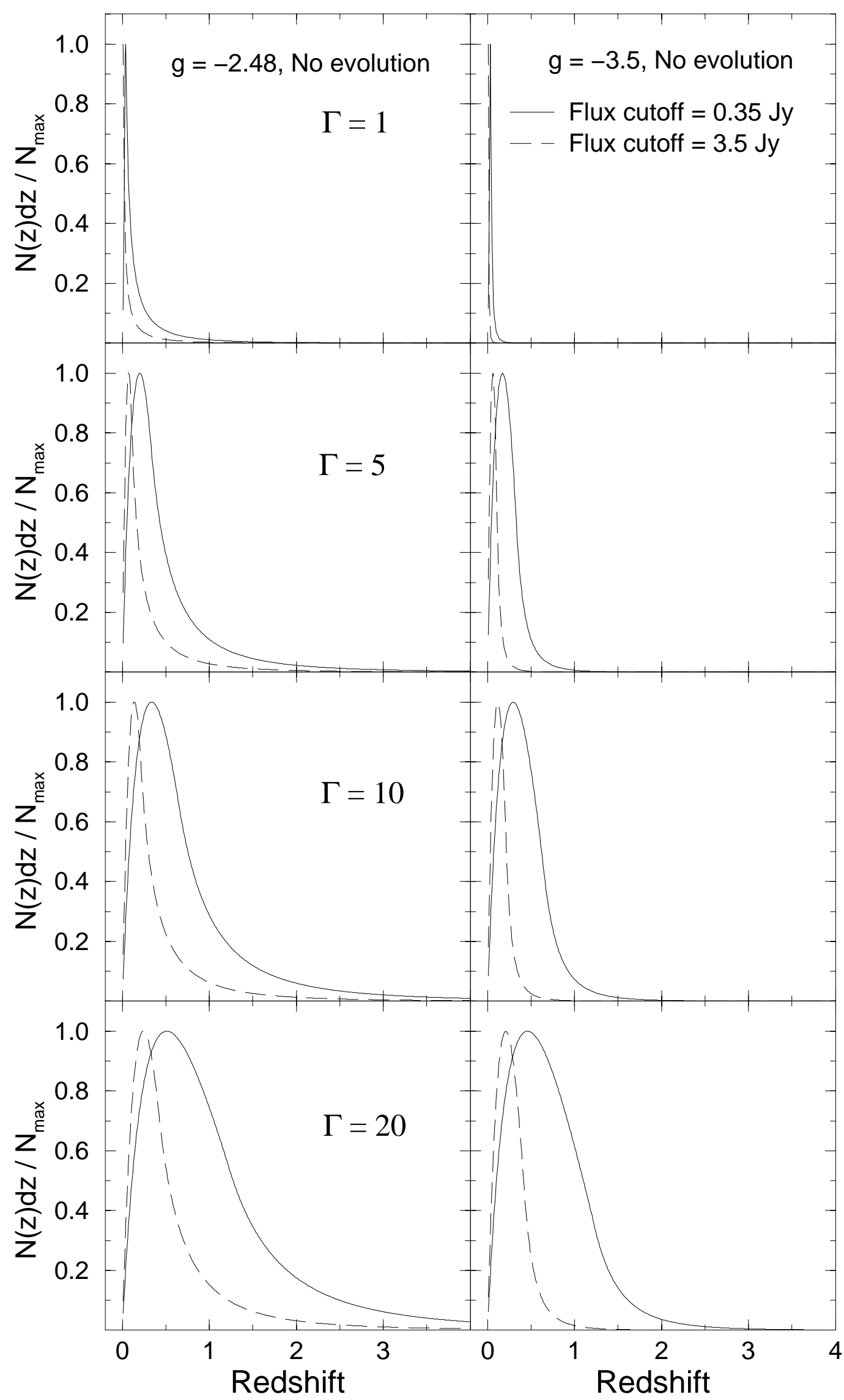

FIG. 1.-Predicted redshift distributions for a canonical jet sample having identical Lorentz factors, according to the $L$ - $\Gamma$-independent (LGI) model with no luminosity evolution described in $\S 2.2$. Each row of models has a different Lorentz factor $(\Gamma)$ as indicated, while the right and left columns have power-law LF slopes of -2.48 and -3.5 , respectively. The LF of the dashed curves has a lower flux cutoff of $3.5 \mathrm{Jy}$, while the solid curves have a cutoff at $0.35 \mathrm{Jy}$. The curves have all been normalized to the same peak value. 


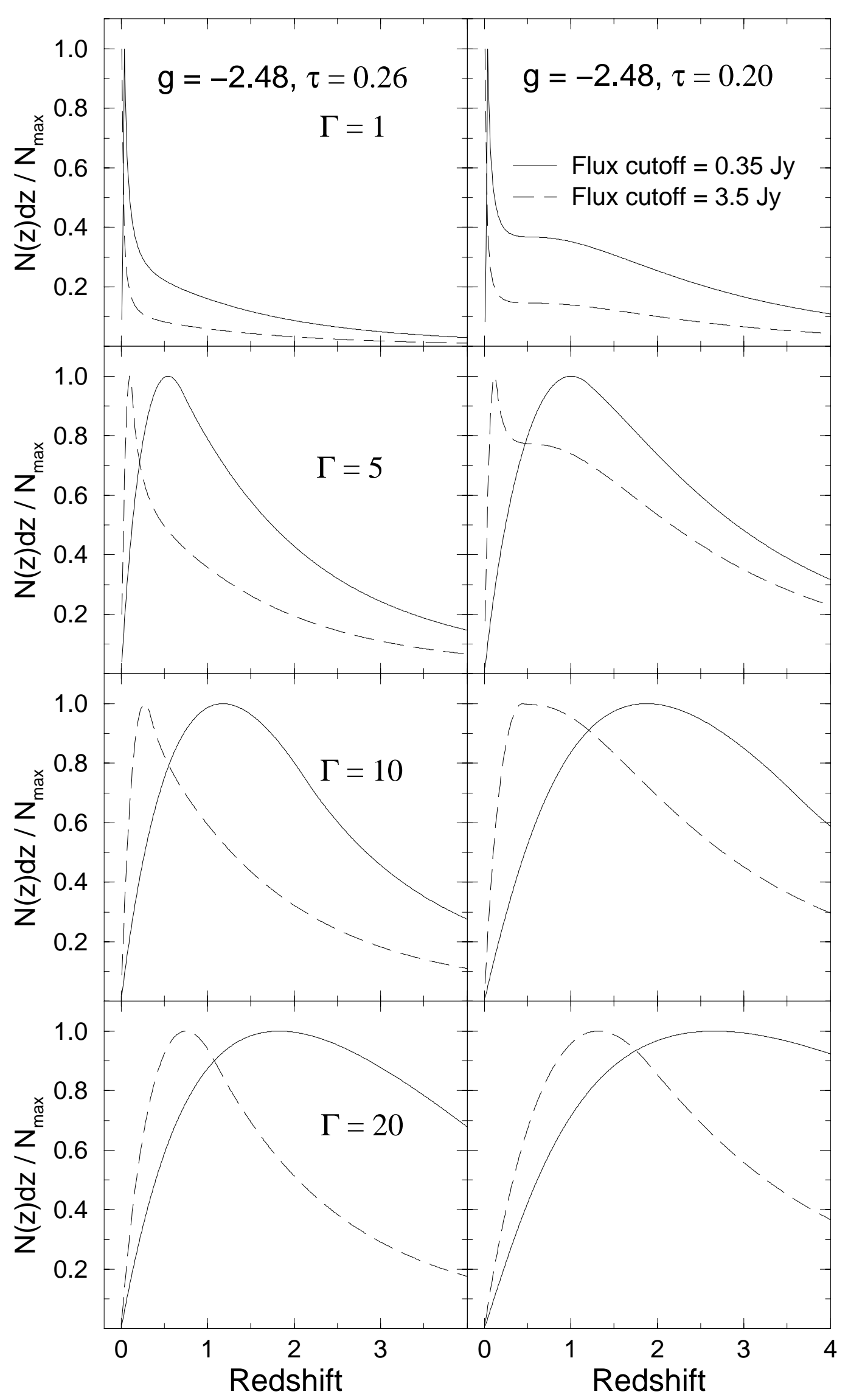

FIG. 2.-Predicted redshift distributions for the LGI model of $\S 2.3$, which includes the effects of luminosity evolution. The LF has power-law slope of -2.48 , and the evolution parameters are $\tau=0.26$ and $\tau=0.2$ for the left and right columns, respectively. The curves have all been normalized to the same peak value. Note the dramatic increase in the relative number of high- $z$ sources when more evolution is added to the model (right column). 

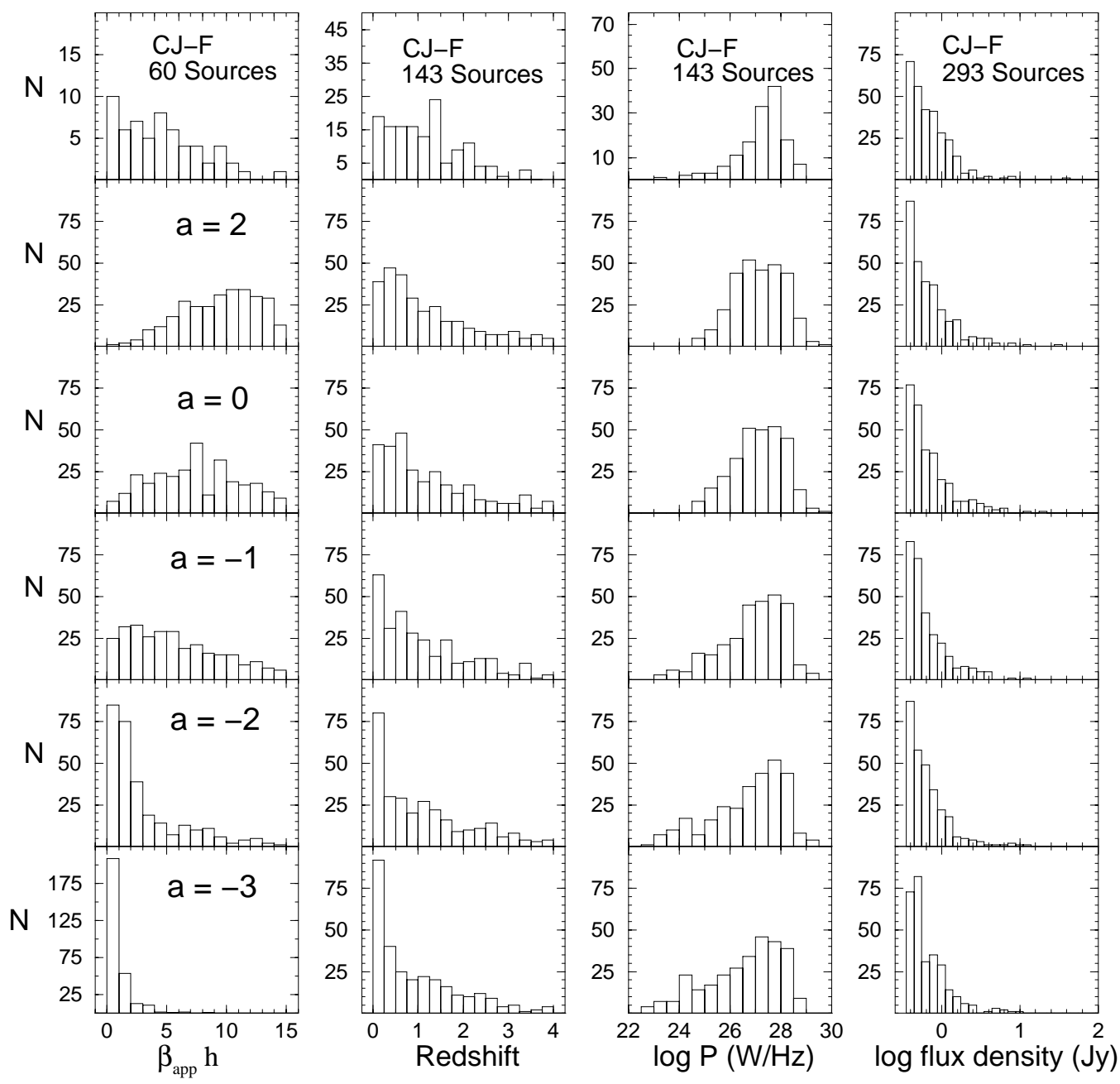

FIG. 3.-Predicted apparent velocity, redshift, monochromatic emitted luminosity $(P)$, and flux distributions for LGI models described in $\S 5.1$, with $N(\Gamma) \propto \Gamma^{a}$. Note the different scale in the lower left-hand panel. In the top panel of each column, we have plotted the current published data for the CJ-F sample. All models were calculated assuming $h=0.65$ and $q_{0}=0$ (see $\S 4.3$ ).

FR II radio galaxy LF as described in $\S 2.3$ (the PULF). This class of object has been postulated as the parent population of powerful quasars (see Urry \& Padovani 1995 for a recent review of unified theories for AGN), which form the bulk of the CJ-F sample.

The properties of our flux-limited samples depend heavily on the low-luminosity cutoff $\left(L_{1}\right)$ of the PULF, which is poorly known. Lower values of $L_{1}$ require a larger parent population to produce 293 sources with flux density past the cutoff, and thus there is an increased chance of extremely rare (per unit volume), highly beamed sources being included in low- $L_{1}$ flux-limited samples.

An estimate for $L_{1}$ can be obtained by examining the source with the lowest monochromatic emitted luminosity $(P)$ in the CJ-F sample. This source $(1146+596 ;$ NGC 3894$)$ has $P_{5 \mathrm{GHz}} \simeq 8 \times 10^{22} h^{-2} \mathrm{~W} \mathrm{~Hz}^{-1}$ and is classified as a radio galaxy. Owing to the Malmquist bias, the lowest $P$ sources in CJ-F are all at low redshifts. Since the volume element is small for low-z objects, these objects are likely to be representative of the most common sources in the parent population and will thus have unbeamed luminosity $L \sim$ $L_{1}$. The low- $P$ sources in the CJ-F sample are also radio galaxies, which, according to current unified models, have viewing angles $\theta \gtrsim 45^{\circ}$ (Urry \& Padovani 1995); this implies a maximum Doppler factor of $2 / \sin \theta$, or $\sim 2.8$.
Under the assumption that $P \propto L \delta^{2}$, this gives a lower limit of $\sim 1 \times 10^{22} h^{-2} \mathrm{~W} \mathrm{~Hz}^{-1}$ for $L_{1}$. We adopt this value for our models and will discuss the impact of raising $L_{1}$ in $\S 5.3$.

\subsection{Lorentz Factor Distributions}

Although it has been shown by Vermeulen (1995) that the parent objects of the CJ-F sample are not likely to have identical Lorentz factors, very little else is known about the intrinsic Lorentz factor distribution of relativistic jets. The best observational constraint on $N(\Gamma)$ is the apparent velocity distribution of the CJ-F sample, which peaks at subluminal speeds and decreases steadily to $\beta_{\mathrm{app}} \simeq 15 \mathrm{~h}^{-1}$. The maximum apparent velocity for a particular Lorentz factor $\Gamma$ is $\beta_{\text {app, } \max }=\left(\Gamma^{2}-1\right)^{1 / 2}$. It has been shown by Cohen (1989) that owing to Doppler beaming, the value of $\beta_{\mathrm{app}, \max }$ for a flux-limited sample will always be very close to that of $\Gamma_{\max }$. Thus, we set $\Gamma_{\max }=15 h^{-1}$ in our models.

The comparison of observed apparent velocity distributions to model predictions is complicated by the possibility that there are differences in the bulk and pattern Lorentz factors of relativistic jets. Numerical hydrodynamic jet simulations by Gómez et al. (1995) have shown that shocks identified with the superluminal components in AGNs can be made to propagate down the jet at a variety of speeds, which depend upon the properties of the initial disturbance 
and other factors. Indeed, there are examples of individual sources that emit superluminal components that have a range of apparent velocities (Vermeulen \& Cohen 1994). We cannot rule out the possibility that the large number of subluminal sources seen to date in VLBI studies may be due to standing shock patterns and other features that have very low measured apparent velocities (see, e.g., Gómez et al. 1995). Only continual observations of the sample being studied can determine the actual statistical significance of this phenomenon.

The exact nature of the correlation between pattern and flow speeds is still not known, owing largely to a lack of reliable estimators of the bulk Lorentz factor of the jet. In this paper, we will concentrate solely on models with equal pattern and bulk Lorentz factors, in order to limit the number of free parameters. The effects of having jets with unequal $\Gamma_{\text {flow }}$ and $\Gamma_{\text {pattern }}$ and their impact on predicted apparent velocity distributions have been studied extensively by Vermeulen \& Cohen (1994).

We have experimented with several other forms of the parent Lorentz factor distribution, including Gaussian and power-law distributions. A Gaussian distribution of Lorentz factors peaked at $\Gamma_{1}$ returns a Gaussian-shaped apparent velocity distribution with peak very close to $\beta_{\text {app }}=\Gamma_{1}$. As this form is inconsistent with the CJ-F data, we have chosen not to discuss further Gaussian $N(\Gamma)$ distributions in this paper.

Power-law forms of $N(\Gamma)$ have been explored by Padovani \& Urry (1992) and produce apparent velocity distributions that depend strongly upon the steepness of the value of the power-law slope. Positive (negative) slopes produce $\beta_{\text {app }}$ distributions that slope upward (downward) toward $\beta_{\text {app }}=\Gamma_{\max }$. The apparent velocity distribution of the CJ-F sample (see Fig. 3) is therefore suggestive of a negative power-law slope for $N(\Gamma)$. In the Monte Carlo simulations presented in $\S 5$, we employ distributions of the form $N(\Gamma) \propto \Gamma^{a}$ and $N(\Gamma) \propto(\Gamma-1)^{a}$, where $a$ is a free parameter.

\subsection{Cosmological Model}

Throughout this paper we adopt the values $h=0.65$ and $q_{0}=0$. For convenience, we include where possible appropriate factors of $h$ in expressions for measured extragalactic quantities. We also include a factor of $h$ in both predicted and observed values of the apparent velocity $\beta_{\text {app }}$ in order to facilitate comparisons with observational data in the literature.

The values of $H_{0}$ and $q_{0}$ are of particular importance to the study of AGNs, as they directly affect the inferred values of Doppler and Lorentz factors we derive from observations of superluminal motions. For example, our choice of upper limit for our Lorentz factor distributions in $\S 4.2$ is strictly dependent on $H_{0}$, and the dependence of volume element on $H_{0}$ in turn affects our predicted redshift distributions as well. We caution therefore that the predictions of our Monte Carlo simulations presented in Figures 3-12 cannot be rescaled in a simple fashion for other values of $H_{0}$.

\subsection{Other Parameters}

As stated previously, the total fluxes of our canonical sources contain no contribution from extended (unbeamed) structure and have spectral indices $\alpha=0$. The sources are assumed to contain two identical, oppositely directed jets, with the jet axis oriented at random with respect to the observer, and the population is randomly distributed with a constant comoving density in the range $0.001 \leq z \leq 4$. Finally, we assume that the intrinsic synchrotron luminosity is independent of $\Gamma$ and all other jet properties (the LGI model). The latter criterion will be dropped in $\S 6$, where we will examine intrinsic $L-\Gamma$-dependent (LGD) models.

\section{MONTE CARLO SIMULATIONS. I. $L-\Gamma-$ INDEPENDENT} (LGI) MODELS

$$
\text { 5.1. } N(\Gamma) \propto \Gamma^{a} \text { Models }
$$

In this section we examine how the predictions of the $N(\Gamma) \propto \Gamma^{a}$ models compare with the observational data of the CJ-F survey for different values of the parameter $a$, which determines the power-law slope of the Lorentz factor distribution.

\subsubsection{Apparent Velocity Distributions}

In Figure 3, we show the $\beta_{\text {app }}$, redshift, monochromatic emitted luminosity, and flux density distributions for models having various values of power-law slope $a$ for the Lorentz factor distribution.

The best fits to the CJ-F apparent velocity data occur for negative values of $a$, since the CJ-F distribution has a downward slope. However, models with very large negative values of $a$ (viz., $a \lesssim-2$ ) contain an insufficient number of high- $\beta_{\text {app }}$ sources. The mean reduced $\chi^{2}$ values for four separate Monte Carlo runs are shown in the top panel of Figure 4, with the standard deviation about the mean value represented by the error bars. The best-fit model occurs for $a=-1.25$, although we do not attach a high significance to this particular value based on fits to the apparent velocity data alone, as they are currently only $\sim 20 \%$ complete for the CJ-F sample. Furthermore, many of the CJ-F sources have been observed at only a few epochs, which raises the possibility that many $\beta_{\text {app }}$ values may be underestimated. This underestimation would steepen the observed $\beta_{\text {app }}$ distribution by moving higher $\beta_{\text {app }}$ sources to smaller $\beta_{\text {app }}$ bins. It is unclear to what extent the CJ-F data are influenced by this effect. We note the case of a well-known source (4C $39.25)$ that was long thought to be stationary until a superluminal component appeared in the 1980s (Marcaide et al. 1985). A handful of other sources (e.g., 3C 273 and 1928+738; Vermeulen \& Cohen 1994 and references therein) have also emitted components having a range of speeds over their observed lifetimes. Such sources may also exist in the CJ-F sample but may not be identified until maps are obtained over a sufficiently large range of epochs.

\subsubsection{Redshift and Monochromatic Emitted Luminosity Distributions}

The CJ-F $z$-distribution is still incomplete, with redshifts currently available for only 192 of 290 sources. It also contains a strong bias, in that the sources with missing redshifts generally have lower than average $5 \mathrm{GHz}$ radio flux densities.

In order to make meaningful comparisons to our models, we have selected a subsample of 184 sources with flux densities $\geq 0.5 \mathrm{Jy}$ from CJ-F. At this flux density cutoff, 143 of these sources have measured redshifts, and the flux density bias is negligible. We compare the redshifts and monochromatic emitted luminosities $(P)$ of this subsample to those sources with flux density $\geq 0.5$ Jy produced by our models using a $\chi^{2}$ test. The CJ-F subsample data are plotted in the 


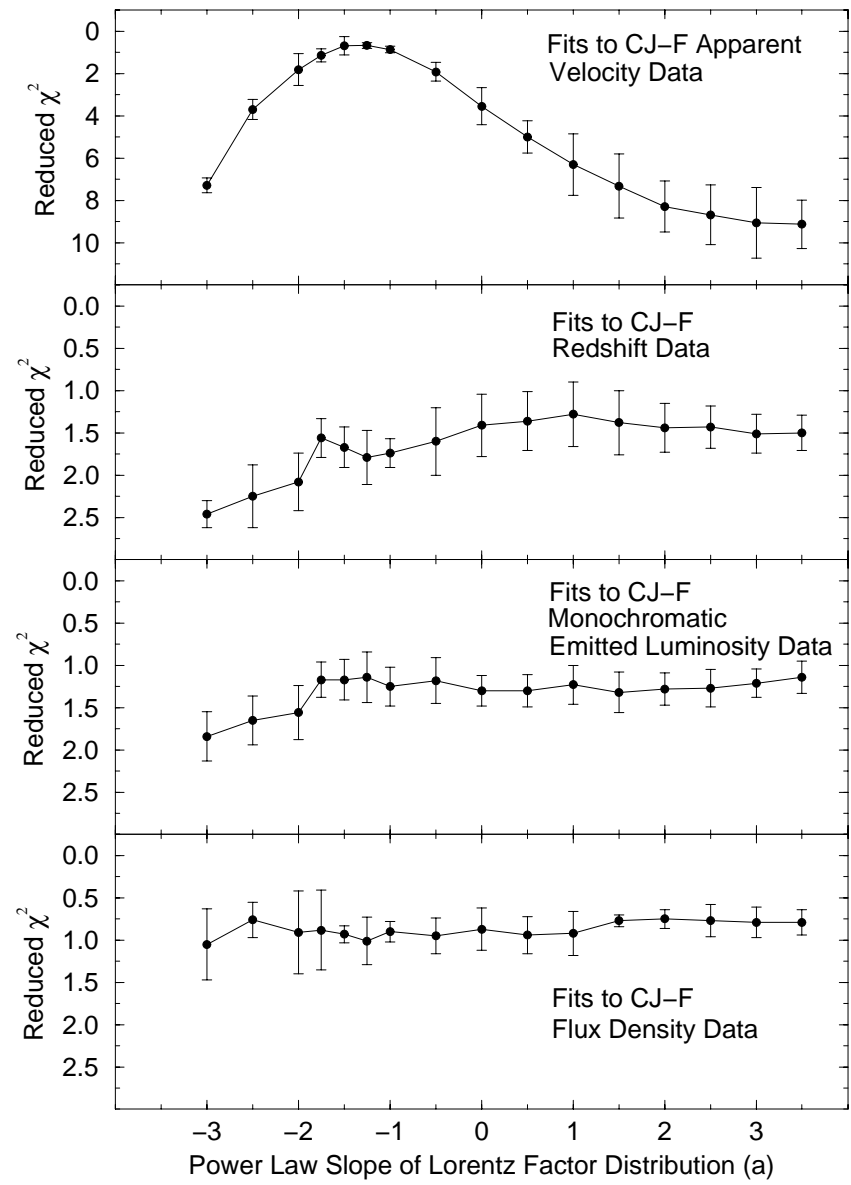

FIG. 4.- Reduced $\chi^{2}$ fit values to the CJ-F apparent velocity, redshift, flux density, and monochromatic emitted luminosity distributions of $L-\Gamma-$ independent (LGI) models, for various values of the Lorentz factor distribution power-law slope $a$ in the LGI model. The values plotted represent the average of four separate Monte Carlo runs, with the standard deviation about the mean value represented by the error bars.

top panels of the second and third columns of Figure 3. The model redshift and $P$ distributions plotted are for complete samples of 293 sources. As the value of $a$ is lowered, the average redshift and monochromatic emitted luminosity of the flux-limited sample steadily decreases. This is due in part to the diminished overall amount of beaming that results when the average Lorentz factor of the parent population is decreased. Also, because of the small volume element at low $z$, the vast majority of low-redshift objects in the positive $a$ models have high Lorentz factors but wide viewing angles and will be sufficiently antibeamed so as to not be included in a flux-limited sample.

The results of our $\chi^{2}$ tests are shown in Figure 4. For the redshift distributions, the variation in $\chi^{2}$ is relatively flat within the range $-2 \leq a \leq 3$, with slightly worse fits indicated for $a<-2$. There is little variation in $\chi^{2}$ for the $P$ distributions in the range $-2 \lesssim a \lesssim 3.5$, with poorer fits for $a \lesssim-2$. The $\chi^{2}$ values for our predicted redshifts and $P$ values are slightly poorer than those associated with the flux density and apparent velocity distributions, which may be due to uncertainties in the evolution parameter of the intrinsic LF. These will be discussed further in $\S 5.3$.

\subsubsection{Flux Density Distributions}

As a further consistency check on our models, we also compare the predicted flux density distributions to the CJ-F data. Although the flux density distribution is essentially a combination of the redshift and monochromatic luminosity distributions, such a test is advantageous as flux densities are available for all sources in the CJ-F sample. The data are plotted using logarithmic bins in the top right-hand panel of Figure 3. The distribution peaks in the first bin and then drops off steeply, with few sources having flux densities greater than $\sim 2 \mathrm{Jy}$. The brightest source in the sample is $3 \mathrm{C}$ 84 , with a $5 \mathrm{GHz}$ flux density of $43 \mathrm{Jy}$.

In general, the predicted flux density distributions provide better fits to the CJ-F data than the fits to the redshift or $P$ distributions (Fig. 4) and do not have a large dependence on the slope of the Lorentz factor distribution.

\subsubsection{Viewing Angle and Doppler Factor Distributions}

The viewing angles to the radio axes of extragalactic radio sources are very difficult to determine observationally, with estimates having been obtained for only a few well-studied sources to date. Nevertheless, since orientation is a major factor in the observed angular extent of a radio source, it is of interest to examine the predicted viewing angle distributions of our models and to discuss the consequences regarding the observed properties of fluxlimited samples. As can be seen in Figure 5 (middle column), the $a \geq-1$ models have a very strong orientation bias, in that the majority of sources have viewing angles $\lesssim 20^{\circ}$. The bias diminishes dramatically with decreasing $a$, along with the average Doppler factor of the sample objects (Fig. 5, right-hand column).

The strong orientation bias found in many of our models appears to be in conflict with the unified model of AGNs (Barthel 1989), which postulates that FR II radio galaxies should be seen at viewing angles $\gtrsim 45^{\circ}$. As the CJ-F sample contains at least 31 radio galaxies, we find models with $a \gtrsim-1.5$ do not predict enough high viewing angle sources to be consistent with the unified scheme.

Another interesting result of our modeling experiments concerns the oft-quoted claim in the literature that a beamed source is typically seen at a viewing angle that maximizes its apparent velocity, namely $\theta_{m}=\arcsin (1 / \Gamma)$. As pointed out by Marscher (1990), there is a high probability that a source will be seen at a value somewhat less than this angle, since the Doppler beaming is maximized for a viewing angle of zero. Vermeulen \& Cohen (1994) find that for a single-valued Lorentz factor population, the most likely angle is roughly $50 \%$ smaller than $\theta_{m}$.

An examination of the viewing angle distributions in our models shows that this is also the case for a population with a distribution of Lorentz factors. In Figure 6, we plot the viewing angle distribution for the $a=-1.75$ model, expressed as a percentage difference between $\theta$ and $\theta_{m}$. The peak of the distribution does not occur at zero but rather at $\sim-40 \%$, and several sources are seen at viewing angles substantially greater than $\theta_{m}$. We find the overall form and median value $(\sim-30 \%)$ of this distribution to be independent of the value of $a$. This result implies that $\theta_{m}$ is not a good indicator of the true viewing angle for objects selected on the basis of beamed flux, especially those that are expected to have modest to large viewing angles, e.g., radio galaxies.

\subsubsection{P versus $\beta_{\text {app }}$ Relation}

Vermeulen (1995) has plotted apparent velocity versus monochromatic emitted luminosity $\left(P=L \delta^{(2+\alpha)}\right)$ for those sources in the CJ-F sample with measured $\beta_{\text {app }}$ 's and finds a 

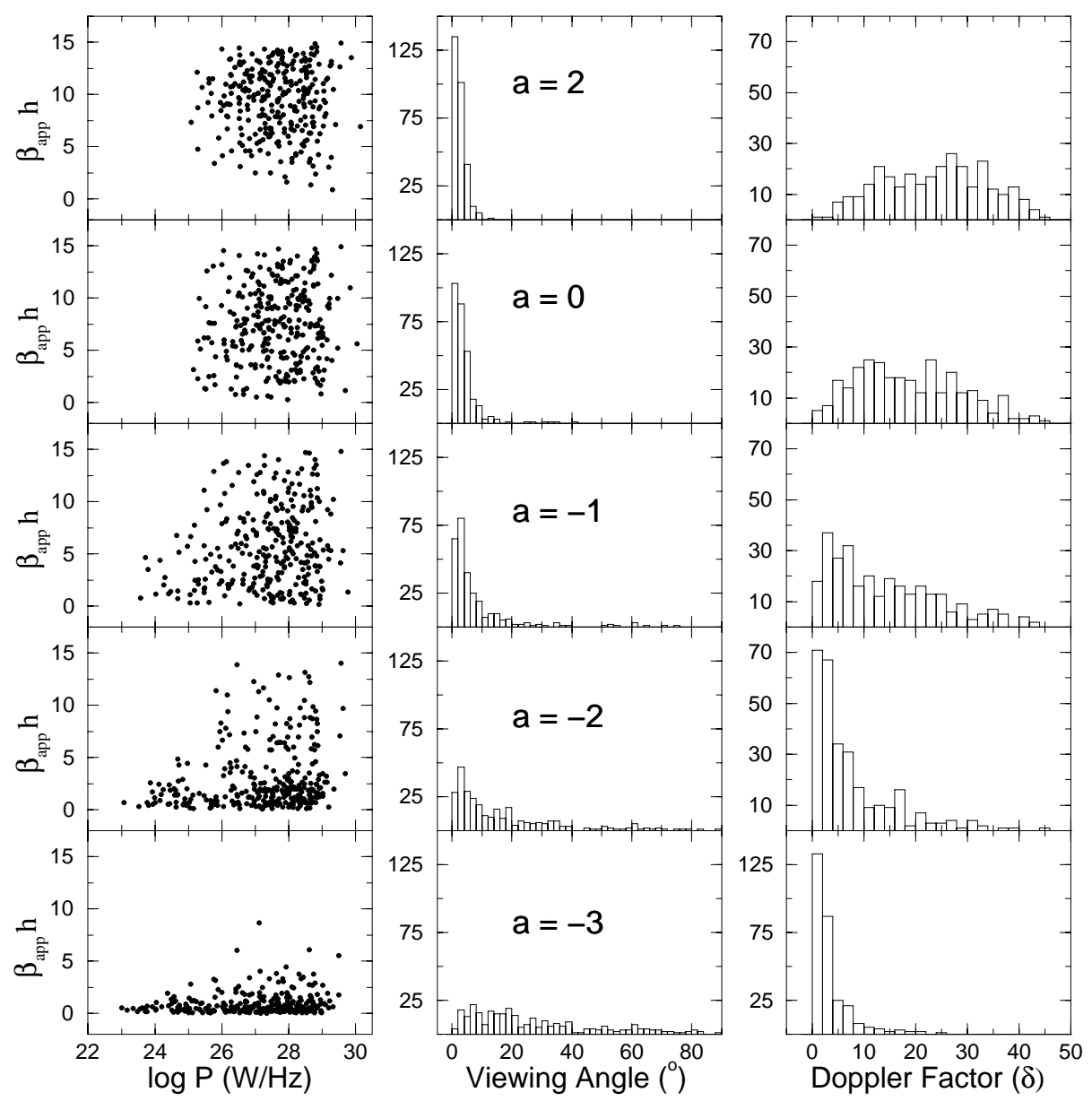

FIG. 5.-Predicted apparent velocity vs. monochromatic emitted luminosity (beamed power : $P$ ), viewing angle, and Doppler factor distributions for LGI models described in $\S 5.1$, with $N(\Gamma) \propto \Gamma^{a}$. Note how the $a \leq-1$ models contain a population of low- $\beta_{\text {app }}$, low- $P$ objects, consistent with those seen in the CJ-F sample. The $a$ parameter also effectively controls the amount of orientation bias in the model, as indicated by the viewing angle and Doppler factor distributions. All models were calculated assuming $h=0.65$ and $q_{0}=0$ (see $\S 4.3$ ).

rising upper envelope to the distribution. In general, there appears to be a lack of sources that have both high apparent velocities and low $P$. This deficit is a direct result of the Malmquist bias and the statistical properties of relativistic beaming.

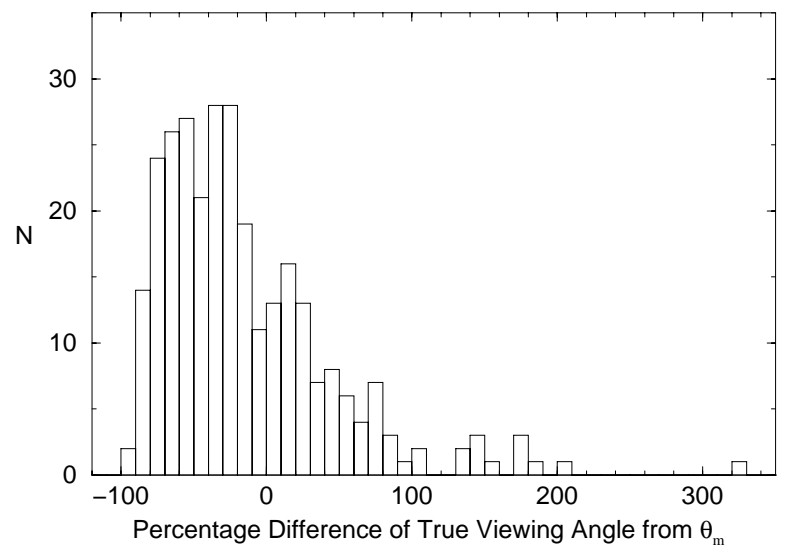

FIG. 6.-Distribution of percentage difference between actual viewing angle and the viewing angle at which the apparent velocity is a maximum $\left[\theta_{m}=\arcsin (1 / \Gamma)\right]$, for an LGI model with $N(\Gamma) \propto \Gamma^{-1.75}$. The majority of sources in a flux-limited sample selected on the basis of beamed flux density are seen at viewing angles substantially less than $\theta_{m}$. The form of this distribution is independent of the power-law slope of the $N(\Gamma)$ distribution.
To understand how this deficit is created, we examine what properties a high- $\beta_{\text {app }}$, low- $P$ source found in a fluxlimited sample must have. First, to have a high apparent velocity, it must have a high Lorentz factor, and a small viewing angle, near $\theta_{m}$. Second, it must have a low redshift in order to have a flux density greater than the sample cutoff. Such a source is extremely rare in a parent population with a Lorentz factor distribution weighted heavily toward low values. This is due to the fact that at low redshifts, the volume element is small; thus the low-redshift sources in the parent population are most likely to have the high-probability characteristics of high viewing angles, low Lorentz factors, and therefore small apparent velocities.

If, however, the Lorentz factor distribution of the parent population is not as heavily weighted toward low values of $\Gamma$ (i.e., $a \gtrsim-1$ ), then the low-redshift population will contain more high Lorentz factor objects, the majority of which will be antibeamed owing to their high viewing angles. However, there will be a few of these low- $z$ sources with small viewing angles that will be relativistically boosted enough to make it into a flux-limited sample (by virtue of their higher Lorentz factors). Therefore, in these models, the lowest $P$ sources are likely to have much higher apparent velocities and smaller viewing angles than the $a \lesssim$ -1 models. Since these sources are not seen in the current CJ-F data, our models are constrained to values of $a$ less than $\sim-1$. We are currently unable to determine best-fit 
values to the $P$ versus $\beta_{\text {app }}$ relation using, for example, a two-dimensional Kolmogorov-Smirnov test, as the apparent velocity data for the CJ-F sample have not yet been published in tabular form.

$$
\text { 5.1.6. Variability Timescales }
$$

The analytical shocked-jet models for quasar and BL Lac variability (see, e.g., Marscher \& Gear 1985; Hughes, Aller, \& Aller 1989) involve a disturbance propagating down the jet at or near the jet speed. If we identify this disturbance with superluminal components seen in VLBI maps, it is possible to define a variability timescale for the canonical jets in our models.

Observations of the ionization parameter in the broad emission line regions of AGNs have shown it to be nearly constant over a large range of luminosity, from Seyfert 1 galaxies to FR II quasars (Weedman 1986, p. 178). This parameter is proportional to $L R^{-2}$, where $L$ is the luminosity and $R$ is the size scale of the ionized region. We assume that a similar expression holds for the scale of a jet; this relation corresponds to the basic structures of both the emission-line region and the jet being scale invariant. We can then construct a simple expression for the variability timescale of our canonical jets. Under this assumption, the variability timescale is $t_{\mathrm{var}} \propto L^{1 / 2}(1+z) \sin \theta / \beta_{\mathrm{app}}$; hence, high- $L$ objects vary on slower timescales owing to their larger intrinsic sizes, at least before the effects of Doppler blueshifts are included.

There are several apparent trends with $t_{\mathrm{var}}$ for our predicted flux-limited samples. First, there is a dearth of rapidly varying sources at high redshift owing to the Malmquist bias, which preferentially selects larger objects at high redshift. Second, although lower $\beta_{\text {app }}$ objects generally have slower variability timescales, there are nevertheless a handful of low-redshift, low- $\beta_{\text {app }}$ sources in our samples that vary rapidly owing to their small $L$ values and therefore small sizes. Third, there is a strong trend of increasing $t_{\mathrm{var}}$ with viewing angle, which is in keeping with the unified model, as well as current data that indicate that radio galaxies on average have slower variability timescales than quasars (Teräsranta \& Valtaoja 1994). Finally, the models display the expected downward trend of faster $t_{\mathrm{var}}$ with increasing Doppler factor, yet there is a large scatter to this distribution. The most rapidly varying sources have Doppler factors in the range $15 \lesssim \delta \lesssim 46$. However, we find that a rapid variability timescale does not necessarily guarantee a large Doppler factor, provided that size scales with luminosity, as the source may just be intrinsically small.

\subsubsection{Size of Parent Population}

The size of the parent population required to produce 293 sources with flux densities greater than $350 \mathrm{mJy}$ is a strong function of $a$, as shown in Figure 7. A Lorentz factor distribution that is skewed toward high values allows for greater Doppler beaming among a larger fraction of sources and thus requires a smaller parent population.

The total comoving volume covered by the CJ-F survey (given a sky coverage of $\sim 3.6 \mathrm{sr}$, out to $z=4$ ) is approximately $825 \mathrm{Gpc}^{3}$, using $h=0.65$ and $q_{0}=0$. Our predicted value of the parent population space density, for example, according to the $a=-1.75$ model is $5.9 \times 10^{-5} \mathrm{Mpc}^{-3}$, which is roughly $14 \%$ of the observed space density for $L_{*}$ elliptical galaxies (Marzke et al. 1994), which are candidates for AGN host galaxies.

We note that our predicted densities are highly sensitive to the parameters of the intrinsic LF, particularly the lowluminosity cutoff $L_{1}$ and the evolution parameter $\tau$. For example, increasing $L_{1}$ by a factor of 10 to $1 \times 10^{23} h^{-2} \mathrm{~W}$ $\mathrm{Hz}^{-1}$ for an $a=-1.75$ model results in a space density that is roughly a factor of 26 smaller. Likewise, increasing the amount of evolution by changing $\tau$ from 0.26 to 0.16 in the $a=-1.25$ model decreases the predicted space density roughly sixfold.

\subsection{Lorentz Factor Distributions of the Form $N(\Gamma) \propto(\Gamma-1)^{a}$}

We also investigate this particular form of the Lorentz factor distribution, as the kinetic energy-likely to be the physical quantity of interest - of a relativistic jet is proportional to $\Gamma-1$.

We assume a range $1.00125<\Gamma<15 h^{-1}$ for the Lorentz factors of the parent population. The lower limit to $\Gamma$ is taken to be the velocity of the clouds in the broad-line region of quasars $(\beta \simeq 0.05)$. A jet with velocity less than this value would most likely have insufficient ram pressure to break free of the emission line region.

Although this form is similar to the $N(\Gamma) \propto \Gamma^{a}$ model at high values of $\Gamma$, the latter model levels off as $\Gamma \rightarrow 1$, while the $(\Gamma-1)^{a}$ form continues to increase when $a<0$. We find very few differences in the predictions of these two models, other than the best-fit value of $a$, which lies in the range $-1 \lesssim a \lesssim-0.5$. In all other respects, the predicted

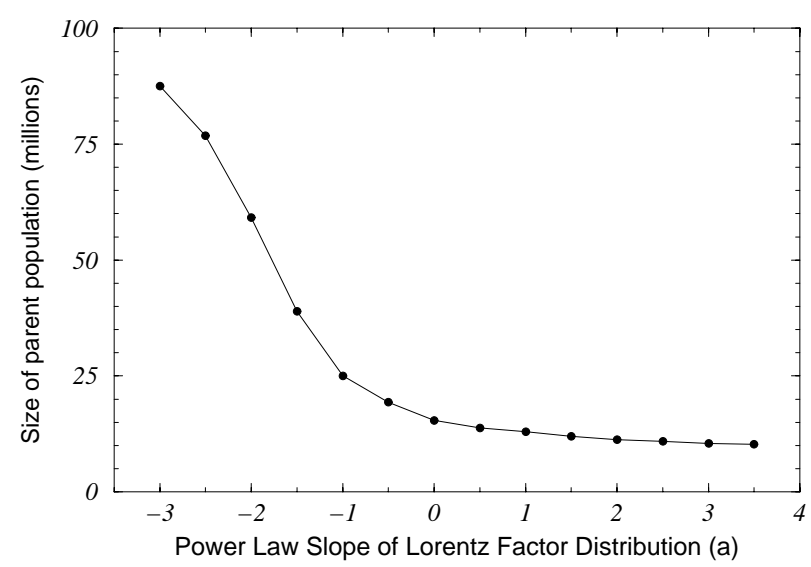

FIG. 7.-Total size of parent population required to produce 293 objects with flux densities $\geq 0.35$ Jy plotted against the power-law slope of the Lorentz factor distribution $a$, where $N(\Gamma) \propto \Gamma^{a}$. 
properties of the flux-limited samples are identical. Values of $a \gtrsim-0.5$ do not reproduce the trend of $\beta_{\text {app }}$ versus $P$ and predict viewing angle distributions that are in conflict with the unified model. As in the case of the previous model, values of $a$ that are too steep $(a \lesssim-1)$ do not create sufficient numbers of high $\beta_{\text {app }}$ sources.

\subsection{Uncertainties in the Intrinsic Luminosity Function}

The intrinsic LF we use for our models, which is based on that of Padovani \& Urry (1992), contains large uncertainties in three parameters: the postbreak slope $\left(G_{2}=\right.$ $-3.9 \pm 0.7)$, the evolution parameter $(\tau=0.26$, with $1 \sigma$ uncertainty range $[0.16,1.0]$ ), and the low-luminosity cutoff $L_{1}$. In $\S 2$ we showed that, for the single-valued Lorentz factor model, changing the LF slope and evolution parameters has a significant impact on the predicted redshift distributions. In order to investigate whether this is also the case for the models of Figure 3, we have computed the distributions of several models using LF parameters within the error ranges given above, with $N(\Gamma) \propto \Gamma^{-1.75}$. Our results are as follows:

1. The LF parameters have very little effect on the predicted $\beta_{\text {app }}$ distributions of the models. For the LGI model, we find the overriding factor in determining the $\beta_{\text {app }}$ distribution in a flux-limited sample to be the distribution of Lorentz factors in the parent population. In a related study, Vermeulen \& Cohen (1994) found that in models in which the pattern and bulk flow Lorentz factors are different, the $\beta_{\text {app }}$ distribution is strongly sensitive to the product of LF slope and Doppler-boosting index. This sensitivity is small, however, in the case (as in our models) where $\Gamma_{\text {flow }}$ and $\Gamma_{\text {pattern }}$ are identical.

2. The postbreak slope $G_{2}$ has little effect on the predicted flux or redshift distributions, since the majority of the sources in the flux-limited samples have intrinsic (unbeamed) luminosities $<L_{\text {break }}$. This is simply a reflection of the shape of the intrinsic LF, in which the ratio of $L_{\text {break }}$ to the low-luminosity cutoff is large and the prebreak slope is steep (-2.48). Intrinsically luminous sources in the parent population are thus very rare and are not likely to have high Doppler factors in models with negative $a$ values. Very few of these sources make it into our predicted flux-limited samples.

3. We find that the evolution parameter has a slight impact on the predicted flux distributions and a very large impact on the predicted redshift and $P$ distributions. This is evident in Figure 8, where we show these distributions for $N(\Gamma) \propto \Gamma^{-1.75}$ models in which we have varied the evolution parameter $\tau$ within the range $0.16 \leq \tau \leq 0.36$.

4. The value of the low-luminosity cutoff of the parent LF $\left(L_{1}\right)$ has a strong effect on the nature of flux-limited samples, since an intrinsically brighter parent population requires a smaller amount of relativistic beaming. In Figure 9 we plot three $a=-1.75$ models with successively higher values of $L_{1}$. A higher $L_{1}$ value produces a flux-limited sample that has, on average, smaller Doppler factors, higher $P$ values, and higher redshifts. The flux density distribution (not shown), on the other hand, is not strongly affected by $L_{1}$. We have also indicated on Figure 9 the size of the parent population, and we note that it decreases dramatically as $L_{1}$ is increased.

\subsection{Summary of LGI Models}

Our Monte Carlo simulations have shown that the fits to the CJ-F data are highly sensitive to the power-law slope (a) of the intrinsic Lorentz factor distribution. We find general consistency with the CJ-F data for $a$ values in the range $-1.75 \lesssim a \lesssim-1.5$, for the form $N(\Gamma) \propto \Gamma^{a}$, or, alternatively $-0.5 \lesssim a \lesssim-1$ for the form $N(\Gamma) \propto(\Gamma-1)^{a}$. Values
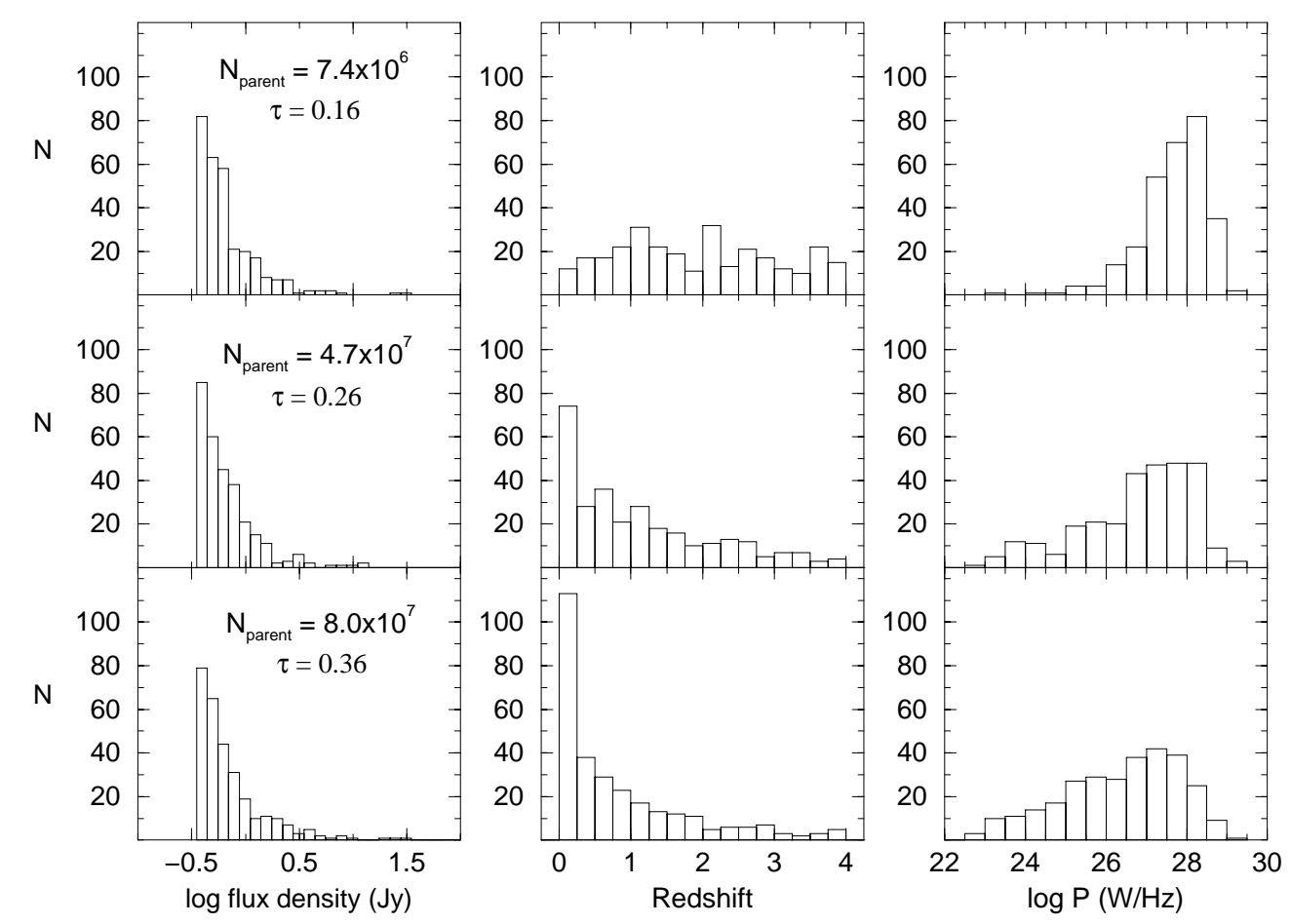

FIG. 8.-Illustration of the effects of the LF evolution parameter $\tau$ on the predicted flux density, redshift, and monochromatic emitted luminosity distributions of the LGI model for $a=-1.75$. Note that changing $\tau$ has a much larger effect on the redshift and $P$ distributions than on the flux density distributions. 

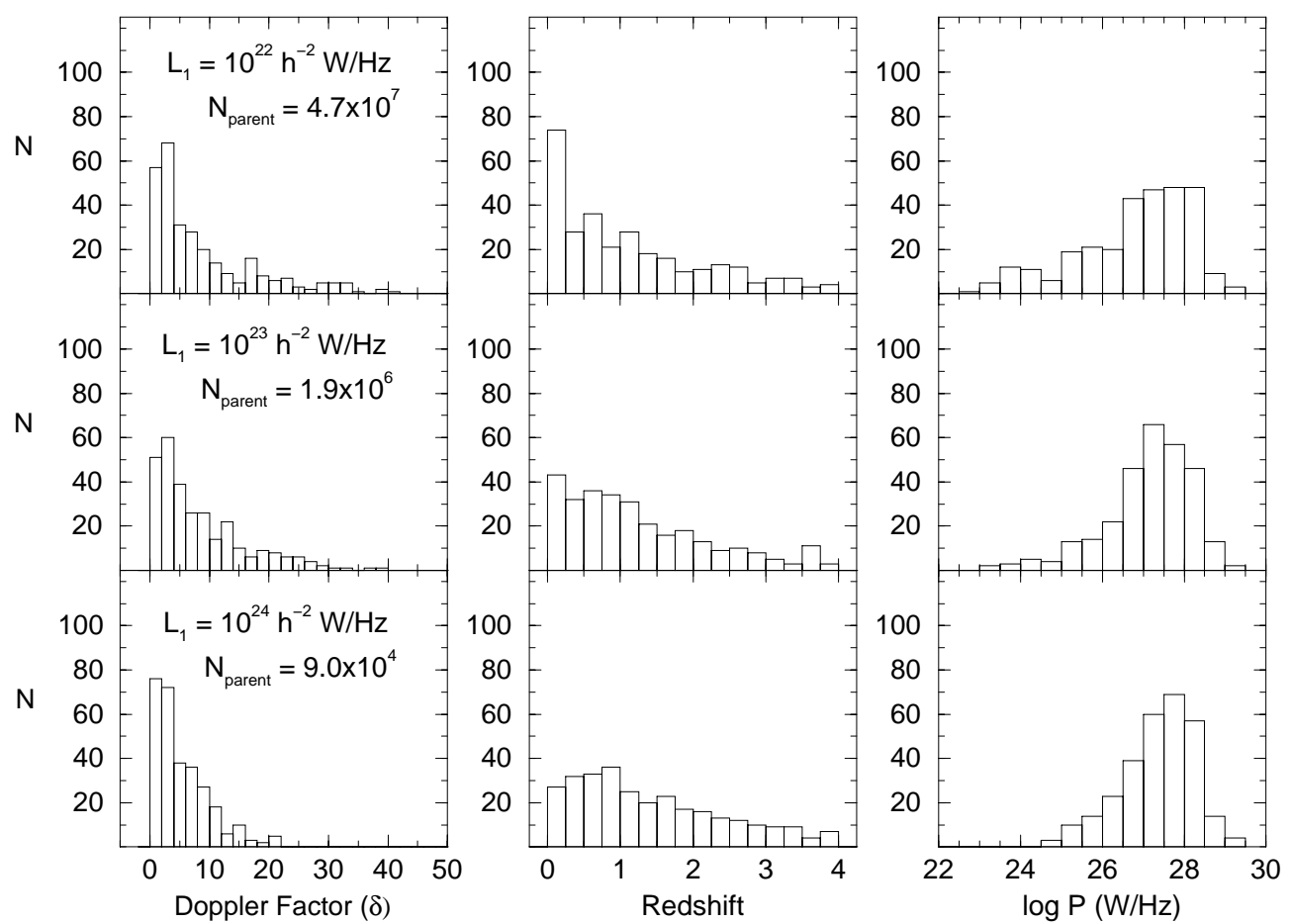

FIG. 9.-Illustration of the effects of changing the low-luminosity cutoff $L_{1}$ in the parent LF for the LGI model with $a=-1.75$. As $L_{1}$ is raised, the redshift distribution flattens, while the Doppler factor distribution steepens to smaller values. The $P$ distribution also steepens slightly toward higher values.

within these ranges yield the best fits to the CJ-F apparent velocity distribution. Furthermore, consistency with the observed $P$ versus $\beta_{\text {app }}$ relation and the unified model give the added constraints $a \lesssim-1$ and $a \lesssim-1.5$, respectively, for the $N(\Gamma) \propto \Gamma^{a}$ models. We do not find the flux density distribution to be a strong constraint on $a$, as it is not highly affected by the form of the Lorentz factor distribution.

Our fits to the CJ-F redshift and $P$ distributions yield slightly poorer reduced $\chi^{2}$ values, in the range $1-2$. The fits to the CJ-F $P$ distribution are especially poor for $a \lesssim-2$, where $N(\Gamma) \propto \Gamma^{a}$. However, our predicted distributions for these parameters are very sensitive to the lower luminosity cutoff and the luminosity evolution of the parent LF, of which there remain large observational uncertainties at present.

Our range of best-fit values for $a$ is slightly higher than the value of $a=-2.3$ obtained by Padovani \& Urry (1992) by fitting the beamed PULF to the observed flat-spectrum quasar luminosity function. However, they used a higher Doppler boost index $(p=2.8)$, which increases the amount of beaming in the population and reduces the need for large numbers of high $\Gamma$ sources.

\section{MONTE CARLO SIMULATIONS. II. $L$ - - -DEPENDENT (LGD) MODELS}

In this section we investigate models in which the bulk Lorentz factor is related to the intrinsic radio luminosity of a source. According to the Blandford \& Rees (1974) jet model based on hydrodynamics, with radiative synchrotron properties given by Marscher (1980), the intrinsic optically thin synchrotron luminosity $L$ is proportional to $\Gamma^{\xi}$ (the results are expected to be similar for magnetohydrodynamics). At present there are very few observational constraints on $\xi$, which depends on the external pressure gradient, intrinsic opening angle, electron energy distribution, and size of the jet. We therefore examine a series of models using a range of $\xi$ values.

\subsection{Unbeamed Luminosity Function}

An important constraint on the LGD models is that the unbeamed LF must match the FR II radio galaxy luminosity function of Padovani \& Urry (1992) (the PULF). If we assume $L=\Gamma^{\xi} \ell$, then the LF has the form

$$
N(L) \propto \int P(\Gamma) P(\ell) \frac{\partial \Gamma}{\partial L} d \ell,
$$

where $\Gamma$ must be expressed in terms of $\ell$ and $L$. Here, $\ell$ is the luminosity for a nonrelativistic $(\Gamma \sim 1)$ jet, and the $P$ 's are probability density functions.

For $P(\Gamma) \propto(\Gamma-1)^{a}$, equation (9) becomes

$$
N(L) \propto \int\left[(L / \ell)^{1 / \xi}-1\right]^{a}(L / \ell)^{1 / \xi} L^{-1} P(\ell) d \ell .
$$

As can be seen from this equation, for arbitrary values of $\xi$ and $a$, there is no suitable function $P(\ell)$ that can adequately reproduce a double power law for $N(L)$ of the form dictated by the PULF. However, a very close approximation to the PULF can be obtained if we use $P(\Gamma) \propto \Gamma^{a}$. In this case, equation (9) becomes

$$
N(L) \propto L^{(a+1) / \xi-1} \int \ell^{-(a+1) / \xi} P(\ell) d \ell,
$$

where the limits of the integral are functions of $L$.

Let us assume a two power-law distribution for $P(\ell)$ with low-luminosity cutoff at $\ell=\ell_{1}$, break at $\ell=\ell_{\mathrm{br}}$, and power-law slopes $g_{1}$ and $g_{2}$ before and after the break.

For the case $\xi>0, N(L)$ has a power-law slope $(a+1) /$ $\xi-1$ in the region $L<\ell_{\mathrm{br}}$ and has a slightly rounded shape in the region $\ell_{\mathrm{br}}<L<\ell_{\mathrm{br}} \Gamma_{\max }^{\xi}$, where $\Gamma_{\max }=15 h^{-1}$ in our models. In the region $L>\ell_{\mathrm{br}} \Gamma_{\max }^{\xi}$, the slope is $g_{2}$. We 
therefore set $\ell_{\mathrm{br}}=L_{\mathrm{br}} / \Gamma_{\max }^{\xi}, \ell_{1}=L_{1}, g_{1}=G_{1}, g_{2}=G_{2}$, and constrain $a=\left(G_{1}+1\right) \xi-1$, where $L_{\mathrm{br}}, L_{1}, G_{1}$, and $G_{2}$ are the parameters of the PULF.

For $\xi<0$, the resulting unbeamed LF has a power-law slope $(a+1) / \xi-1$ in the region $L<\ell_{1}$, a slope approximately equal to $g_{1}$ for $\ell_{1}<L<\ell_{\mathrm{br}}$, and slope $g_{2}$ for $L>\ell_{\mathrm{br}}$. We set the low-luminosity cutoff to $L_{1} / \Gamma_{\max }^{\xi}$, since the range of $L$ extends down to $L=\ell_{1} \Gamma_{\text {max }}^{\xi}$. We also set $\ell_{\mathrm{br}}=L_{\mathrm{br}}, g_{1}=G_{1}$, and $g_{2}=G_{2}$, and add the constraint $a=\left(G_{1}+1\right) \xi-1$.

\subsection{Negative $\xi$ Models}

The general effect of introducing a negative correlation between intrinsic luminosity and Lorentz factor is to reduce the effects of Doppler bias in a flux-limited sample, since the highly boosted sources will now have lower intrinsic luminosities. However, the effect is counteracted somewhat by the constraints on the parent LF. If $\xi$ is made more negative, then the Lorentz factor distribution must be biased toward higher values (by increasing $a$ ), thus increasing the amount of beaming in the sample. In this section we investigate the possible differences in the predicted properties of flux-limited samples based on this $L-\Gamma$-anticorrelated (LGA) model and the LGI models of $\S 5$.

We begin by comparing the case of $(\xi=-2.7, a=3)$ to a corresponding LGI model with $(\xi=0, a=3)$. The most important distinction between the LGA and LGI models is that the former predicts many more low-Lorentz factor sources in the final flux-limited sample. This has an impact on the Doppler factor and apparent velocity distributions, which are both shifted toward lower values (Fig. 10).

The redshift distribution of the LGA model is steeper than the LGI, with the highest redshift sources in the LGA model having $z \lesssim 3$. The $P$ distribution is also shifted toward slightly lower values in the LGA model (Fig. 10).

The apparent velocity distribution of the LGA model (Fig. 10) has a negative slope despite the highly positive slope of the parent $N(\Gamma)$ distribution. Thus both the $\xi$ and $a$ parameters can have a large impact on the predicted apparent velocity distributions of flux-limited samples.

In contrast to the LGI model, which contains no source with viewing angle $\gtrsim 15^{\circ}$, the $\xi=-2.7$ model contains several low- $\delta$ sources that have viewing angles ranging from $15^{\circ}$ to $90^{\circ}$. However, it does not predict the same trend
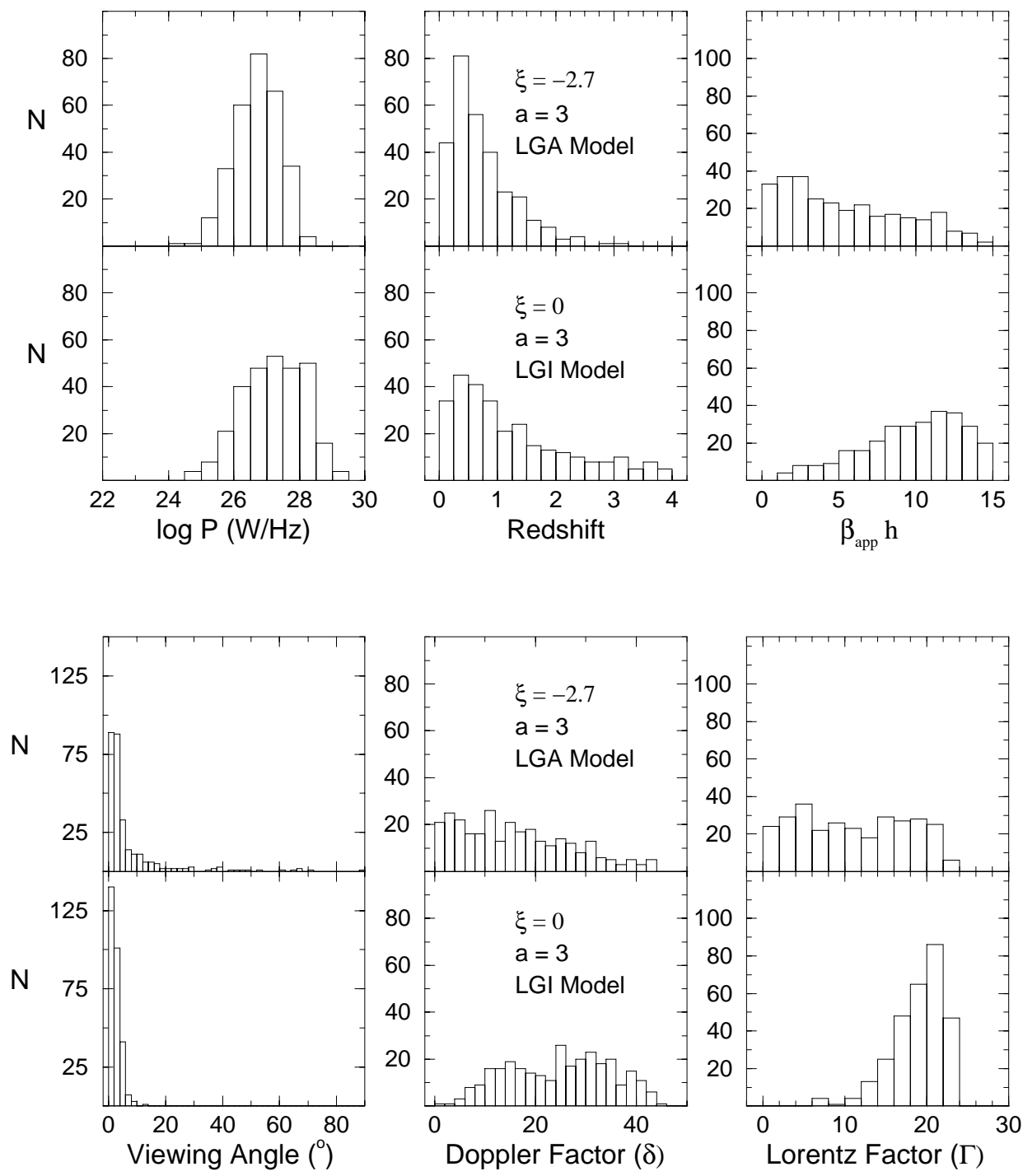

FIG. 10.-Predicted distributions for the $L-\Gamma$-anticorrelated (LGA) model with $\xi=-2.7$ (top panels) and $\xi=0$ (LGI; bottom panels), for identical Lorentz factor distributions of power-law slope $a=3$. Note the differences in the Lorentz factor and apparent velocity distributions associated with the LGA and LGI models. 
between $P$ and $\beta_{\text {app }}$ as seen in the CJ-F sample. This is due to the fact that very few high-redshift, high-Lorentz factor sources are predicted, since (1) their intrinsic luminosities are lower due to the $\xi$ parameter and (2) their flux densities are lower owing to their large distances. Given that the Malmquist bias dictates that high-redshift sources have high $P$ 's, there is a downward trend of $\Gamma$ (and consequently $\beta_{\text {app }}$ ) with $P$. This trend, which is inconsistent with the CJ-F data, occurs only for models with $\xi \lesssim-2.5$. In the case of models with greater $\xi$ values, the Doppler bias dominates over the inverse-square law dimming, so that there is no trend between $\Gamma$ and redshift.

The LGA models in the range $-2.5 \lesssim \xi<0$ do not reproduce the $P$ versus $\beta_{\text {app }}$ trend of CJ-F either, since they do not predict a sufficient number of low- $P$ sources. This is directly attributable to the increased amount of beaming that results from using positive values of $a$. On the other hand, the faintest sources in the $(\xi>0)$ and $(\xi=0, a<0)$ models are roughly 2 orders of magnitude fainter than those of the LGA model and are more consistent with the lowest $P$ sources in the CJ-F sample.

\subsection{Positive $\xi$ Models}

As was the case with the negative $\xi$ models, in order to match the prebreak slope of the PULF, the Lorentz factor distributions of this class of model are constrained to have power-law slope $a=-1.48 \xi-1$. Again we assume $N(\Gamma) \propto \Gamma^{a}$ for all models. We will refer to this model henceforth as the luminosity- $\Gamma$ correlated (LGC) model.

Introducing a positive correlation between intrinsic luminosity and Lorentz factor tends to enhance the Doppler bias in a flux-limited sample, but once again the effect is counteracted to a certain extent by the restrictions on the parent Lorentz factor distribution, which in this case must have negative slope. In Figure 11 we plot predicted distributions for models with $\xi=1.01$ and $\xi=0$, where $a=-2.5$.

High-Lorentz factor sources are more heavily favored by the LGC models owing to their higher intrinsic luminosities and maximum Doppler factors. The average Doppler factor for a flux-limited sample is thus higher than the corresponding LGI model, and the average viewing angle is much lower. However, owing to the large numbers of low-
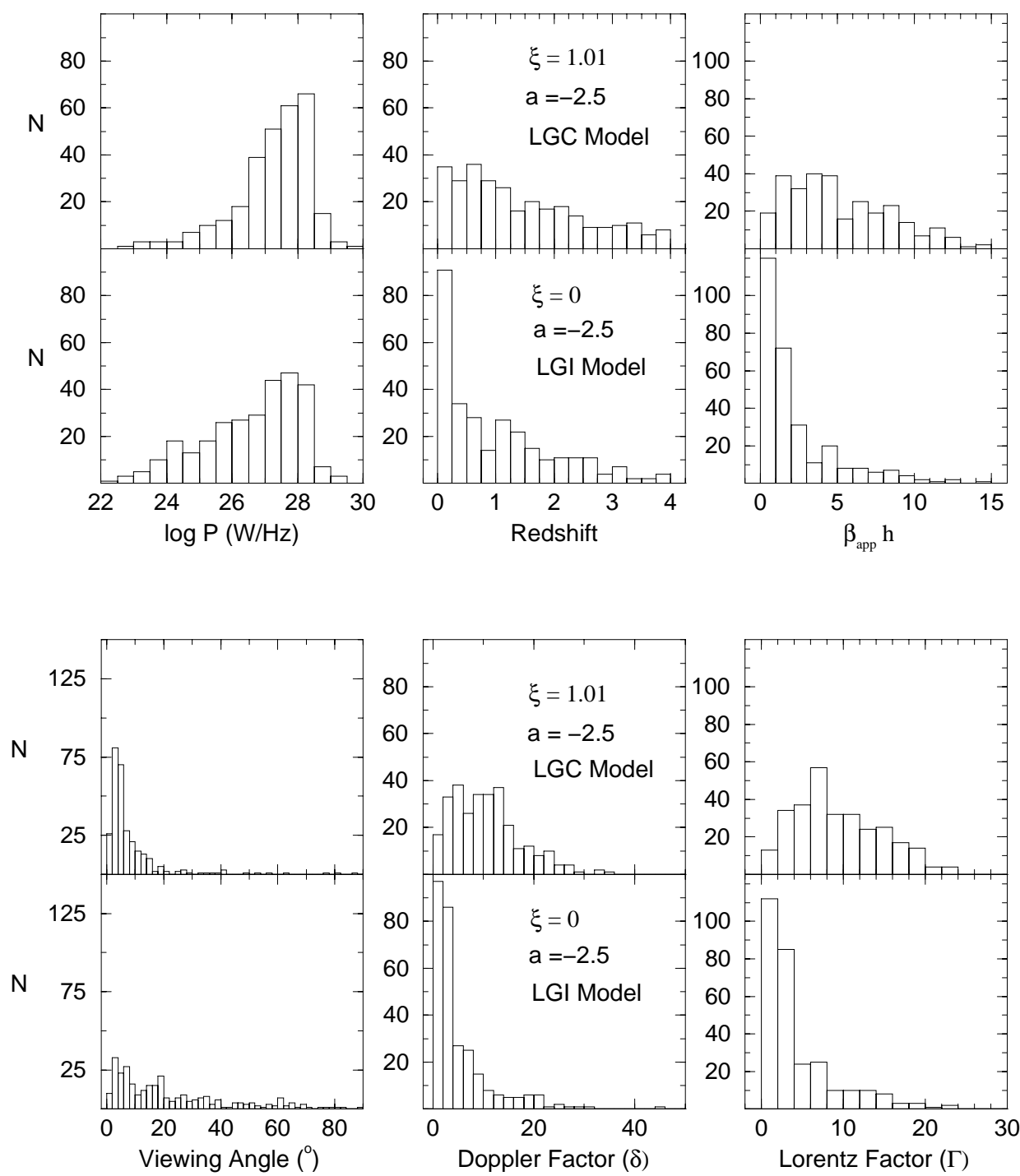

FIG. 11.-Predicted distributions for the luminosity- $\Gamma$ correlated (LGC) model with $\xi=1.01$ (top panels) and $\xi=0$ (LGI; bottom panels), for identical Lorentz factor distributions of power-law slope $a=-2.5$. Note the stronger orientation bias of the LGC model, and the significant flattening of the redshift distribution. 
Lorentz factor objects in the parent population, the LGC model still contains some low- $\Gamma$, low-redshift sources with high viewing angles. There are sufficient numbers of these sources to reproduce the $P$ versus $\beta_{\text {app }}$ relation, but these models on average predict only $\sim 10$ sources with $\theta \gtrsim 45^{\circ}$, which is not consistent with the CJ-F data and the unified scheme. The variability properties of both models are similar, with the one exception that the predicted fluxlimited LGC model sources span a total range of variability timescale roughly 2 orders of magnitude smaller than the LGI model. This is due to the scarcity of long $t_{\mathrm{var}}$, low- $\Gamma$ sources with high viewing angles predicted by the LGC model.

The increased amount of effective beaming in the LGC model also allows sources to be seen at higher redshifts; hence, the much flatter $z$ distribution as compared to the LGI model. The sources in the LGC flux-limited sample also have higher average rest-frame monochromatic emitted luminosities $(P)$ as a result of the Malmquist bias. The apparent velocity distribution of the LGC model is not as steep as the corresponding LGI model, owing to the higher Doppler factors of the sample objects.

There is a large difference in the predicted size of the parent population of the LGI and LGC models, with the latter requiring roughly 9 times fewer parent objects to create the required 293 sources. This is merely a reflection of the effective increase in the monochromatic emitted luminosity of high- $\Gamma$ parent objects that comes from having a positive correlation of synchrotron luminosity with $\Gamma$.

We find the $\xi$ parameter to have little effect on the reduced $\chi^{2}$ values associated with the predicted apparent velocity, redshift, and flux density distributions of the LGC models. However, models in the range $0.7 \lesssim \xi \lesssim 0.3$ provide slightly better fits to the CJ-F monochromatic emitted luminosity distribution.

\subsection{Summary of L- $\Gamma$-dependent Models}

Perhaps the most important constraint on models that involve a relationship between Lorentz factor and synchrotron luminosity is the condition that the unbeamed LF of the population must resemble the PULF. As we have seen, this considerably narrows the range of parameter space that is viable. On the other hand, this constraint on the parent LF makes it very difficult to distinguish between the best-fit
LGC and LGI models, unless the intrinsic Lorentz factor distribution of the parent jet population is accurately known. The main difference in these models lies in sources with high viewing angles, which are predicted to be more common in the LGI models.

We now consider possible ways of discriminating observationally between our best-fit LGI model $\left[N(\Gamma) \propto \Gamma^{-1.75}\right.$, $\xi=0]$ and an LGC model with $N(\Gamma) \propto \Gamma^{-2}, \xi=0.68$. We do not consider the LGA models further, as they do not predict the observed $P$ versus $\beta_{\text {app }}$ trend in the CJ-F sample.

We find very little difference in the $\chi^{2}$ significance of the fits between the above LGI and LGC models. Both models reproduce the $P$ versus $\beta_{\text {app }}$ trend, but the upper envelope of the LGC model is more sharply defined, which is more consistent with the CJ-F data (Fig. 12). These differences can be examined in more detail using two-dimensional Kologorov-Smirnov tests, once the complete $\beta_{\text {app }}$ data for the CJ-F data are obtained. A strong difference also occurs in the predicted Doppler factor distributions, with high $(\delta>20)$ Doppler factor sources (representative of blazars) making up $25 \%$ of the LGC sample but only $13 \%$ of the LGI sample (see Fig. 12).

The predictions of the LGI and LGC models for the variability timescale are similar, with the exception that the total range of variability seen in the LGC model is roughly 2 orders of magnitude smaller owing to the smaller number of objects with high viewing angles. The major distinction of the LGC models as compared to the LGI models is that the median variability timescale of the high $\left(\beta_{\text {app }}>10 h^{-1}\right)$ objects is only $\sim 20$ times faster than the low $\left(\beta_{\text {app }}<\right.$ $2.5 h^{-1}$ ) objects, whereas the LGI models predict a ratio of $\sim 200$.

Finally, we note that if better constraints on the number density and LF of the CJ-F parent population can be obtained, this can provide another constraint on our models, as the best-fit LGI model requires a parent population $\sim 5$ times larger than the best-fit LGC model.

\section{DISCUSSION}

A main goal of this paper has been to determine whether statistical methods can be used to look for evidence of a correlation between Lorentz factor and intrinsic synchrotron luminosity in the CJ-F sample. We have shown, however, that at present, such a correlation is not required
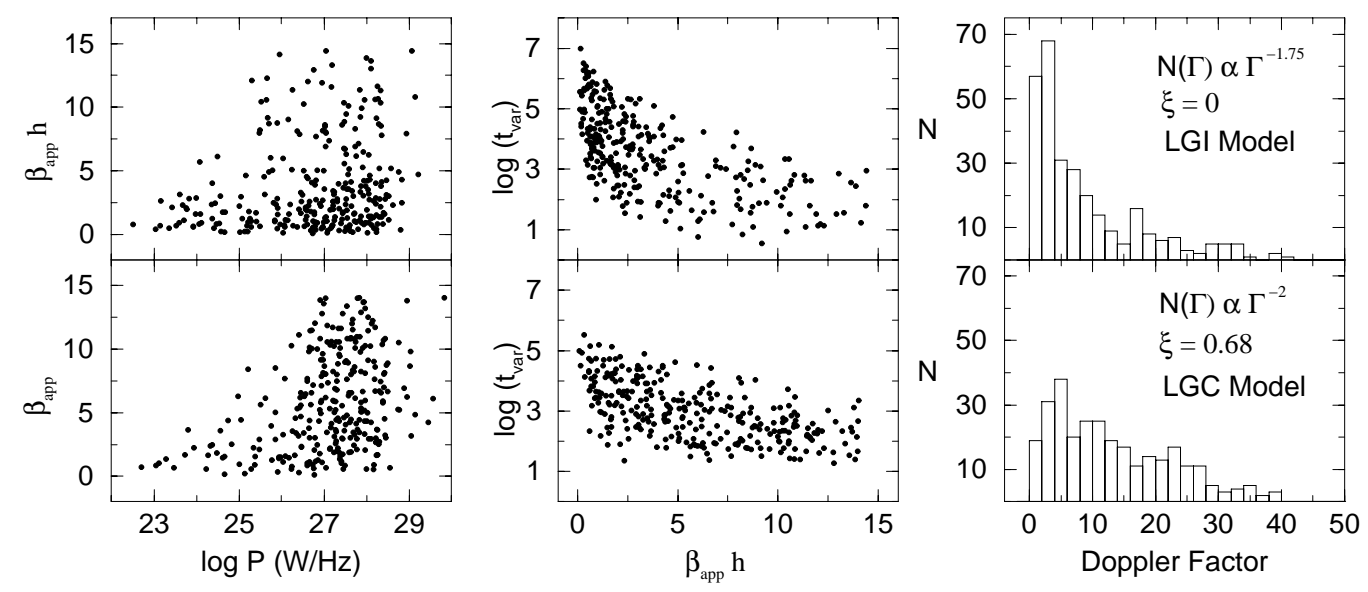

FIG. 12.-Comparison of best-fit LGI models (top panels) and LGC models (bottom panels). The upper envelope of the LGC $P$ vs. $\beta_{\text {app }}$ distribution is better defined and is more consistent with the CJ-F sample data. The LGC model sources span a smaller range of variability timescale (plotted in arbitrary time units) and predict fewer sources with Doppler factors greater than 20. 
for the simple beaming model to reproduce the data. On the other hand, the current incomplete observational knowledge of the parent population of the CJ-F sample makes it very difficult to discriminate between the LGI and LGC models. In particular, the uncertainties associated with the lower cutoff and evolution of the LF make it impossible to use our predicted redshift distributions and number densities by themselves as rigorous constraints. Furthermore, the possibility of large differences between the jet bulk and pattern Lorentz factors may render comparisons between predicted and observed apparent velocity distributions invalid. Our models make distinct predictions for the Doppler factor and viewing angle distributions of the objects in flux-limited samples, but unfortunately these quantities are very difficult to determine observationally. Until more reliable methods are developed to obtain Doppler factors and viewing angles of AGNs, any observational tests that are made must be of a simple nature, so that possible scatter in the observed quantities will not affect the overall result. One such test, which can discriminate between the LGI and LGC models, involves the variability timescales of low and high apparent velocity sources in CJ-F sample.

Another important parameter than can potentially affect the results we have presented in this paper is our choice of Doppler-boosting index $(2-\alpha)$. While this index is valid for continuous jets, time dilation in the observer's frame causes the index to increase to $3-\alpha$ for a single component that has a finite lifetime, such as a relativistic shock. A fluxlimited sample chosen at a single epoch, such as the CJ-F sample, therefore runs the risk of including flaring sources, whose flux densities are dominated by a single shocked component (for which the index $3-\alpha$ should be used). Unfortunately, it is impossible to determine what fraction of sources in the CJ-F sample might fall under this category, as VLBI images of the sample were not taken at the same time as the survey, and extensive monitoring data are only available for a handful of sources. Admittedly, the CJ-F sample may also be less than ideal for comparison to our models in one other respect: some low-redshift sources may have been included on the basis of their extended flux density, despite the spectral flatness criterion and high selection frequency. This problem will be alleviated, however, once VLBI and deep VLA images are obtained for the entire sample.

One of the most important general findings of our study is that a wide range of characteristics of compact radio sources can be the result of the strong selection effects of relativistic beaming combined with the intrinsic relations among physical parameters in a jet. For example, highly variable sources can consist of either highly beamed objects at moderate redshifts or moderately beamed, lowluminosity, low-redshift objects. The former are rapidly variable because of their small sizes - a consequence of the general scaling law: size $\propto L^{1 / 2}$. This exercise has thus shown that extreme behavior of blazars such as rapid variability can paradoxically be explained not as a consequence of strong beaming in all blazar-like objects but rather as a general selection effect resulting from a combination of intrinsic source characteristics (viz., small size), relativistic beaming, and Malmquist bias in the entire population.

\section{CONCLUSIONS}

We have investigated the properties of flux-limited samples of compact extragalactic radio sources in which the synchrotron emission is relativistically beamed. We summarize our findings as follows:

1. The apparent velocity distribution of the CJ-F sample is best fitted by models incorporating a power-law distribution of Lorentz factors. For models in which there is no correlation between intrinsic synchrotron luminosity and Lorentz factor, we obtain good fits using $N(\Gamma) \propto \Gamma^{a}$, where $-1.75 \lesssim a \lesssim-1.5$. We also find good fits using the form $N(\Gamma) \propto(\Gamma-1)^{a}$, where $-1 \lesssim a \lesssim-0.5$.

2. The upper envelope to the CJ-F monochromatic emitted luminosity $(P)$ versus apparent velocity $\left(\beta_{\text {app }}\right)$ distribution (Vermeulen 1995) is reproduced by our Monte Carlo simulations and is a result of the combined selection effects of Malmquist bias and Doppler beaming.

3. Models in which the Lorentz factor and intrinsic synchrotron luminosity are anticorrelated are inconsistent with the CJ-F sample data, as they do not reproduce the $P$ versus $\beta_{\text {app }}$ envelope and do not predict sufficient low- $P$ sources.

4. Models in which there is a positive correlation between $\Gamma$ and intrinsic synchrotron luminosity $(L)$ provide good fits to the CJ-F apparent velocity, monochromatic emitted luminosity, flux density, and $P$ versus $\beta_{\text {app }}$ distributions but predict very few sources with viewing angles greater than $\sim 45^{\circ}$. This appears to be in conflict with the number of radio galaxies seen in the CJ-F sample, which the unified model predicts to be at high viewing angles. These models also predict fewer numbers of high Doppler factor $(\delta>20)$ sources and a smaller range of variability timescale than models that have no correlation between $\Gamma$ and $L$.

5. The parent population of the CJ-F sample is predicted to be very large: on the order of $10^{7}-10^{7.7}$ objects are required to produce 293 objects with flux densities greater than $350 \mathrm{mJy}$ for the best-fit LGC and LGI models, respectively. For $h=0.65$, this translates into space densities of $1.3 \times 10^{-5} \mathrm{Mpc}^{-3}$ and $5.9 \times 10^{-5} \mathrm{Mpc}^{-3}$, which corresponds to approximately $3 \%-14 \%$ of the observed space density for $L_{*}$ elliptical galaxies (Marzke et al. 1994).

6. We find consistency with the results of Vermeulen \& Cohen (1994), who determined that for a flux-limited sample of compact radio sources with identical Lorentz factors, the most likely viewing angle is roughly half of $\theta_{m}$, the angle at which a source's apparent velocity is maximized. Our simulations that employ both a range of Lorentz factors and a luminosity function show that sources on average have viewing angles $30 \%$ less than $\theta_{m}$ and in some cases up to $300 \%$ more than this estimate. In general, therefore, $\theta_{m}$ is a poor estimator of the true viewing angle of an object selected on the basis of beamed flux density. This finding is illustrative of one of the many complex selection effects associated with flux-limited sampling that must be considered carefully when making general statements about AGN.

7. The present uncertainties associated with luminosity function of the parent population of the CJ-F sample have a strong impact on our predicted number densities, redshifts, and beamed luminosities and thus limit somewhat the usefulness of these quantities as constraints on our models. Improved estimates of the low-luminosity LF cutoff $L_{1}$ and the evolution parameter $\tau$ would provide additional means of distinguishing between the LGI and LGC models.

The large size of the parent population predicted by our models suggests that our knowledge of AGNs thus far has 
been gleaned from detailed studies of an extremely small fraction of the total AGN population. Until considerably deeper surveys of compact radio sources can be completed, it is of great importance to understand fully the complicated selection effects associated with Doppler boosting and Malmquist bias in order to glean as much information as possible regarding the parent population. As more detailed observations of larger flux-limited samples become available, we hope to be able to test some of the predictions presented in this paper and to be in a position to constrain further models of relativistic jets.

The authors wish to thank an anonymous referee for a careful reading of the original manuscript and useful suggestions that greatly improved this paper. The research described in this report was supported in part by the National Science Foundation through grant AST 91-16525.
Barthel, P. D. 1989, ApJ, 336, 606

Blandford, R. D., \& Rees, M. J. 1974, MNRAS, 169, 395

Cohen, M. H. 1989, in BL Lac Objects, ed. L. Maraschi, T. Maccacaro, \& M. H. Ulrich (Berlin: Springer), 13

Dunlop, J. S., \& Peacock, J. A. 1990, MNRAS, 247, 19

Gómez, J. L., Martí, J. M., Marscher, A. P., Ibáñez, J. M., \& Marcaide, J. M. 1995, ApJ, 449, L19

Hughes, P. A., Aller, H. D., \& Aller, M. F. 1989, ApJ, 341, 54

Marcaide, J. M., et al. 1985, Nature, 314, 424

Marscher, A. P. 1980, ApJ, 235, 386 1990, in Parsec-Scale Radio Jets, ed. J. A. Zensus \& T. J. Pearson (Cambridge: Cambridge Univ. Press), 236

Marscher, A. P., \& Gear, W. K. 1985, ApJ, 298, 114

Marzke, R. O., Geller, M. J., Huchra, J. P., \& Corwin, H. G. 1994, AJ, 108, 437

\section{REFERENCES}

Padovani, P., \& Urry, C. M. 1992, ApJ, 387, 449

Taylor, G. B., Vermeulen, R. C., Readhead, A. C. S., Pearson, T. J., Henstock, D. R., \& Wilkinson, P. N. 1996, ApJS, 107, 37

Teräsranta, H., \& Valtaoja, E. 1994, A\&A, 283, 51

Urry, C. M., \& Padovani, P. 1991, ApJ, 371, 60 1995, PASP, 107, 803

Urry, C. M., \& Shafer, R. A. 1984, ApJ, 280, 569

Vermeulen, R. C. 1995, Proc. Natl. Acad. Sci., 92, 11385

Vermeulen, R. C., \& Cohen, M. H. 1994, ApJ, 430, 467

Wall, J. V., \& Peacock, J. A. 1985, MNRAS, 216, 173

Weedman, D. W. 1986, Quasar Astronomy (Cambridge: Cambridge Univ. Press) 